\title{
Host-guest Assembly of H-Bonding Networks in Covalent Organic Frameworks for Ultrafast and Anhydrous Proton Transfer
}

\author{
Xiaowei Wu, ${ }^{\dagger}, \S$ Ziya Liu, ${ }^{\dagger}, \S \mathrm{Hu}$ Guo, ${ }^{\dagger, \S}$ You-lee Hong, ${ }^{\perp}$ Bingqing Xu, ${ }^{*}, \dagger$ Kun Zhang, ${ }^{\dagger}$ Yusuke \\ Nishiyama, ${ }^{\perp}, \uparrow$ Wei Jiang, ${ }^{\dagger}$ Satoshi Horike ${ }^{\dagger}$ Susumu Kitagawa ${ }^{\ddagger}$ and Gen Zhang, ${ }^{*}, \dagger$ \\ ${ }^{\dagger}$ School of Chemical Engineering, Nanjing University of Science and Technology, Nanjing, Jiangsu \\ 210094, China. \\ Institute for Integrated Cell-Material Sciences, Institute for Advanced Study, Kyoto University, \\ Yoshida, Sakyo-ku Kyoto 606-8501, Japan. \\ ${ }^{\perp}$ RIKEN -JEOL Collaboration Center, Tsurumi, Yokohama, Kanagawa 230-0045, Japan. \\ IJEOL RESONANCE Inc., 3-1-2 Musashino, Akishima, Tokyo 196-8558, Japan. \\ ${ }^{\S}$ X. W, Z. Liu and H. G contributed to this work equally. \\ This work dedicates to Professor Susumu Kitagawa on the occasion of his 7oth birthday. \\ *E-mail: bingqingxu@njust.edu.cn (B.X.); zhanggen@ njust.edu.cn (G.Z.)
}




\section{Materials and general methods}

All chemicals and solvents are commercially available, and used without further purification. All solvents were dried and distilled according to conventional methods.

Powder X-ray diffraction (PXRD): PXRD patterns were collected on a Rigaku MiniFlex with $\mathrm{CuK} \alpha$ radiation.

Fourier transform infrared (FT-IR): IR spectrum was measured on a Bruker Optics ALPHA FT-IR spectrometer with Universal ATR accessory between the ranges of 4000 to $500 \mathrm{~cm}^{-1}$.

Solution nuclear magnetic resonance (NMR): Liquid state ${ }^{1} \mathrm{H} /{ }^{13} \mathrm{C} /{ }^{19} \mathrm{~F} /$ nuclear magnetic resonance spectroscopy were collected on a Bruker Avance III instrument with AS500 magnet equipped with a cryoprobe $(500 \mathrm{MHz})$.

Electrospray ionization mass spectrometry (ESI-MS): ESI mass spectra were collected on a Bruker model micrOTOF II using MeCN as a solvent with an ionization temperature of $150^{\circ} \mathrm{C}$.

Water contact angle (WCA): The water contact angles were measured ongoniometer (JC2000C, Japan) equipped with video capture.

Solid-state nuclear magnetic resonance (ssNMR): All experiments were measured using a JEOL JNM-ECZ600R spectrometer at $14.01 \mathrm{~T}$ with ${ }^{1} \mathrm{H}$ resonance frequency of 599.7 MHz, equipped with a $2 \mathrm{~mm}$ double resonance MAS probe (JEOL RESONANCE Inc, Japan). 1D ${ }^{1} \mathrm{H}$ spectra and ${ }^{1} \mathrm{H} T_{2}$ relaxation time were measured using single pulse and spin-echo pulse sequence, respectively, at $20 \mathrm{kHz}$ spinning rate in the temperature range of $0-100{ }^{\circ} \mathrm{C}$. $1 \mathrm{D}{ }^{13} \mathrm{C}$ and $2 \mathrm{D}{ }^{1} \mathrm{H}-{ }^{13} \mathrm{C}$ spectra were measured using cross polarization magic angle spinning (CPMAS) and heteronuclear correlation (HETCOR) sequence. ${ }^{13} \mathrm{C}$ spectra were recorded at MAS of $20 \mathrm{kHz}$ and recycle delay was $4.2 \mathrm{sec}$ for COF-F6 and $2.0 \mathrm{sec}$ for COF-F6-IL. RF field strength of ${ }^{1} \mathrm{H}$ and ${ }^{13} \mathrm{C}$ nuclei was $166.7 \mathrm{kHz}$ at the $\pi / 2$ pulse width of $1.5 \mu$ and $164.3 \mathrm{kHz}$ at the $\pi / 2$ pulse width of 1.52 $\mu \mathrm{s}$, respectively. $1 \mathrm{D}$ and $2 \mathrm{D}{ }^{1} \mathrm{H}$ double-quantum-single-quantum (DQ-SQ) spectra were measured using R $122_{2}^{5}$ pulse sequence. The spectra were processed with the Delta software (JEOL RESONANCE Inc).

Scanning electron microscope (SEM): The SEM images were collected using a Hitachi S-3000N system.

Gas adsorption: Gas adsorption measurement $\mathrm{N}_{2}$ adsorption measurements were performed on a BELSORP II mini. $\mathrm{H}_{2} \mathrm{O}$ adsorption measurements were performed on a BELaqua3 (BEL-Japan, Inc.). Samples were activated at $120^{\circ} \mathrm{C}$ for $16 \mathrm{~h}$ before the 
measurement.

Thermogravimetric analysis (TGA): TGA was performed using a RigakuTG8120 under flowing $\mathrm{N}_{2}$ with $10 \mathrm{~K} \mathrm{~min}^{-1}$ ramp rate. Samples were heated in a Platinum pan $\left(900{ }^{\circ} \mathrm{C}, 10{ }^{\circ} \mathrm{C} \min ^{-1}\right)$ under a $\mathrm{N}_{2}$ flux $\left(60 \mathrm{~mL} \mathrm{~min}^{-1}\right)$.

AC impedance measurements: Impedance results of COFs powders were collected and analyzed by Solartron 1260A frequency response analyzer. The powders were degassed at $100{ }^{\circ} \mathrm{C}$ under vacuum for $12 \mathrm{~h}$ in advance. Then powders (ca. $20 \mathrm{mg}$ ) were pressed into pellets at $500 \mathrm{~kg}$ for 5 minutes by a $5 \mathrm{~mm}$ die and sandwiched between two stainless steel electrodes under Ar atmosphere (in a glove box). The measurements were performed over a frequency range from $1 \mathrm{MHz}$ to $1 \mathrm{~Hz}$ with a voltage amplitude of 30 $\mathrm{mV}$ from 40 to $140{ }^{\circ} \mathrm{C}$ inside an $\mathrm{Ar}$ filled glove box. Impedance data were fitted by equivalent circuit simulation to obtain resistance values using ZView software. The proton conductivity was calculated by Equation S1.

Equation S1. $\sigma=\mathrm{L} /(\mathrm{Z} \times \mathrm{A})$. (where L is thickness of the pellet $(\mathrm{cm})$, A is the electrode area $\left(\mathrm{cm}^{2}\right)$, and $\mathrm{Z}$ is the impedance $\left.(\Omega)\right)$

\section{Synthetic procedures}

Ionic liquid (dema)( $\left.\mathrm{H}_{2} \mathbf{P O}_{4}\right)$ and (dema)(HSO 4$)$ were synthesized according to reported procedure. ${ }^{[1]}$ COF-C2 (COF-42 in Ref 2) were synthesized following the reported method. ${ }^{[2]}$

Scheme S1. Synthesis of 2,5-Bis(hexyloxy)terephthalohydrazide (Mono-C6).

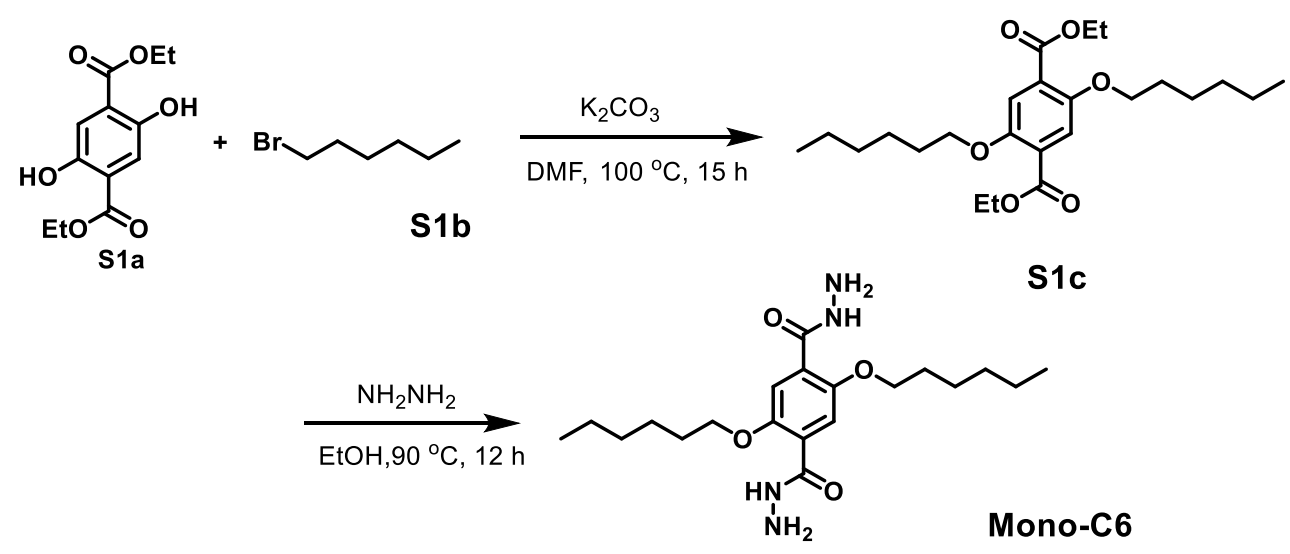

2,5-Bis(hexyloxy)terephthalohydrazide (Mono-C6): S1c was synthesized according to the reported method (Org. Lett. 2004, 6, 229). $1.09 \mathrm{~g}$ (2.0 mmol) of S1c was dissolvd in $15 \mathrm{ml}$ of ethanol and $2 \mathrm{ml}$ of hydrazine hydrate. The mixture was stirred and heated to reflux for $12 \mathrm{~h}$. After cooling, white crystals precipitated, which were isolated by filtration, and recrystallization with ethanol. The white crystal was then dried to obtain 
the final product Mono-C6 as white solid (0.93 g, 90\% yield). ${ }^{\mathbf{1}} \mathbf{H}$ NMR (500 MHz, $\left.\mathrm{CDCl}_{3}\right): \delta 9.20(\mathrm{~s}, 2 \mathrm{H}), 7.85(\mathrm{~s}, 2 \mathrm{H}), 4.18(\mathrm{dd}, J=8.3,4.7 \mathrm{~Hz}, 8 \mathrm{H}), 1.99-1.80(\mathrm{~m}, 4 \mathrm{H})$, $1.47(\mathrm{dd}, J=14.3,6.7 \mathrm{~Hz}, 4 \mathrm{H}), 1.42-1.30(\mathrm{~m}, 8 \mathrm{H}), 0.92(\mathrm{t}, J=6.6 \mathrm{~Hz}, 6 \mathrm{H}) .{ }^{13} \mathbf{C} \mathbf{N M R}$ (125 MHz, $\mathrm{CDCl} 3): \delta=165.5,151.0,123.1,115.7,72.6,38.0,31.9,31.8,31.7,29.6$, $29.3,26.8,22.7,22.6,14.1$.

Scheme S2. Synthesis of 2,5-bis(3,3,4,4,4-entafluorobutoxy)terephthalohydrazide (Mono-F4)<smiles>CCOC(=O)c1cc(O)c(C(=O)OCC)cc1O</smiles><smiles>O=C(O)C(F)(F)C(F)(F)CCO</smiles><smiles>CCOC(=O)c1cc(OCCC(F)(F)C(F)(F)F)c(C(=O)OCC)cc1OCCC(F)(F)C(F)(F)F</smiles>
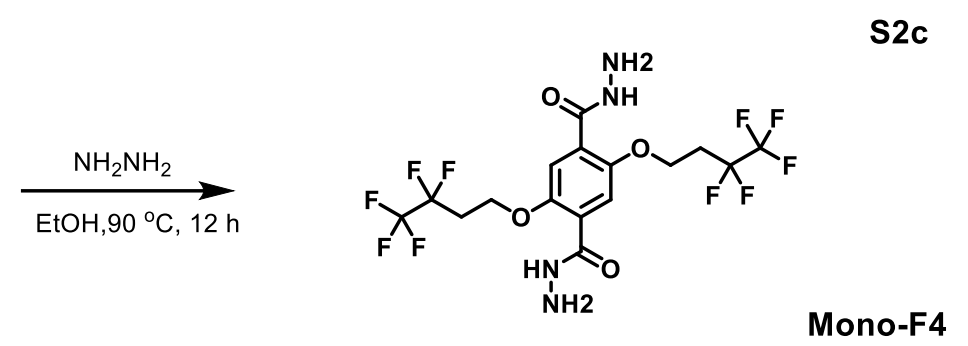

Diethyl 2,5-bis(3,3,4,4,4-pentafluorobutoxy)terephthalate (S2c): S2c was synthesized according to the reported literature with a modified procedure (J. Am. Chem. Soc. 2020, $142,14357)$. Diisopropyl azodicarboxylate $(1.56 \mathrm{~mL}, 8.0 \mathrm{mmol})$ was added to a mixture of diethyl 2,5-dihydroxyterephthalate S1a (1.02 g, $4.0 \mathrm{mmol})$ and triphenylphosphine $(2.08 \mathrm{~g}, 8.0 \mathrm{mmol})$ in tetrahydrofuran $(20 \mathrm{~mL})$, and the mixture was stirred at room temperature for $30 \mathrm{~min}$ under argon atmosphere. Then $\mathbf{S 2 b}(1.313 \mathrm{mg}, 8.0 \mathrm{mmol})$ was added to the solution and the solution was stirred and heated to reflux under argon atmosphere for 3 days. The reaction mixture was evaporated under reduced pressure, and the residue was purified by column chromatography on silica gel (dichloromethane/methanol 40/1 to 20/1) to give the title compound S2c as white solid (1.64 g, 75\% yield).

2,5-bis(3,3,4,4,4-pentafluorobutoxy)terephthalohydrazide (Mono-F4). 1.09 g (2.0 mmol) of S2c was dissolved in $15 \mathrm{~mL}$ of ethanol and $2 \mathrm{~mL}$ of hydrazine hydrate. The mixture was stirred and heated to reflux for $12 \mathrm{~h}$. After cooling to room temperature, 
the suspension was filtered, and the residue was recrystallized from ethanol to afford Mono-F4 as colorless crystals (0.88 g, 85\% yield). ${ }^{1} \mathbf{H}$ NMR $\left(500 \mathrm{MHz}, \mathrm{CDCl}_{3}\right) \delta 7.57$ (s, 2H), $\delta 7.41(\mathrm{~s}, 2 \mathrm{H}), 4.42(\mathrm{q}, 4 \mathrm{H}), 2.81(\mathrm{~m}, 4 \mathrm{H}) .{ }^{13} \mathbf{C} \mathbf{N M R}\left(125 \mathrm{MHz}, \mathrm{CDCl}_{3}\right): \delta=$ $170.18,168.02,155.97,152.57,122.92,122.20,114.57,64.48,33.02 .{ }^{19}$ F NMR (470 $\left.\mathrm{MHz}, \mathrm{CDCl}_{3}\right) \delta-85.8,-117.4$.

Scheme S3. Synthesis of 2,5 -bis $((3,3,4,4,5,5,6,6,6-$ nonafluorohexyl)oxy)terephthalohydrazide (Mono-F6).<smiles>CCOC(=O)c1cc(O)c(C(=O)OCC)cc1O</smiles>

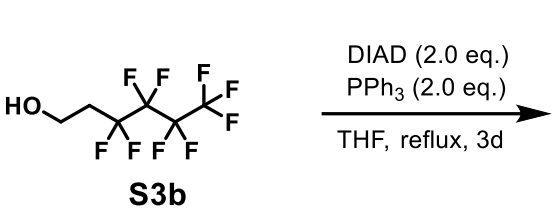<smiles>CCOC(=O)c1cc(OCCC(F)(F)C(F)(F)C(F)(F)F)c(C(=O)OCC)cc1OCCCC(F)(F)C(F)(F)C(F)(F)C(F)(F)F</smiles><smiles>NC(=O)c1cc(OCCC(F)(F)C(F)(F)C(F)(F)F)c(C(=O)N[Na])cc1OCCC(F)(F)C(F)(F)C(F)(F)C(F)(F)F</smiles>

Diethyl 2,5-bis((3,3,4,4,5,5,6,6,6-nonafluorohexyl)oxy)terephthalate (S3c): S3c was synthesized according to the reported literature (J. Am. Chem. Soc. 2020, 142, 14357) Diisopropyl azodicarboxylate $(1.56 \mathrm{~mL}, 8.0 \mathrm{mmol})$ was added to a mixture of diethyl 2,5-dihydroxyterephthalate $\mathbf{S 1 a}(1.02 \mathrm{~g}, 4.0 \mathrm{mmol})$ and triphenylphosphine (2.08 g, 8.0 $\mathrm{mmol})$ in tetrahydrofuran $(20 \mathrm{~mL})$, and the mixture was stirred at room temperature for 30 min under argon atmosphere. Then $\mathbf{S 3 b}(1.29 \mathrm{~mL}, 8.0 \mathrm{mmol})$ was added to the solution and the solution was stirred and heated to reflux under argon atmosphere for 3 days. The reaction mixture was evaporated under reduced pressure, and the residue was purified by column chromatography on silica gel (dichloromethane/methanol 40/1 to 20/1) to give the title compound as white solid (1.85 g, $2.48 \mathrm{mmol}, 62 \%$ yield).

2,5-Bis((3,3,4,4, 5, 5, 6, 6, 6-nonafluorohexyl)oxy)terephthalohydrazide (Mono-F6). 1.49 $\mathrm{g}(2.0 \mathrm{mmol})$ of $\mathbf{S 3 c}$ was dissolved in $15 \mathrm{~mL}$ of ethanol and $2 \mathrm{~mL}$ of hydrazine hydrate. The mixture was stirred and heated to reflux for $12 \mathrm{~h}$. After cooling to room temperature, the suspension was filtered, and the residue was recrystallized from ethanol to afford Mono-F6 as colorless crystals (1.19 g, $1.60 \mathrm{mmol}, 80 \%$ yield). 


\section{Scheme S4. Synthesis of COF-C6}

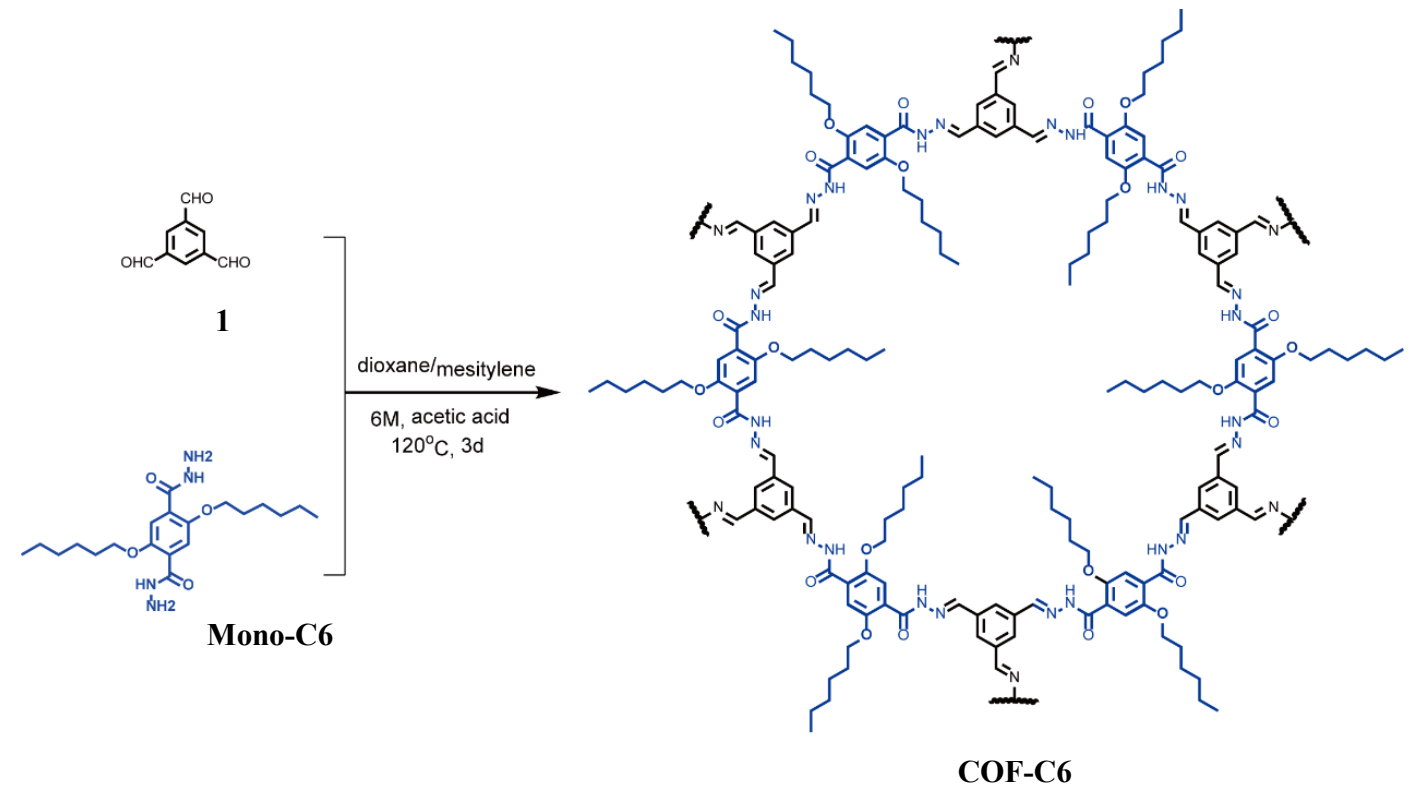

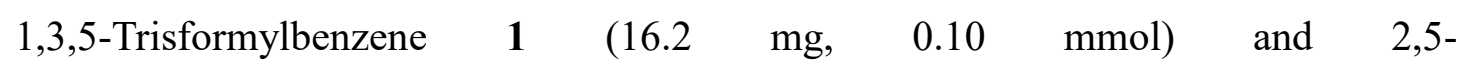
bis(hexyloxy)terephthalohydrazide Mono-C6 (59.1 mg, $0.15 \mathrm{mmol}$ ) were weighed into a glass ampoule (volume of ca. $20 \mathrm{~mL}$, body length of $18 \mathrm{~cm}$, neck length of $9 \mathrm{~cm}$ ). To the mixture was added 1,4-dioxane $(1.0 \mathrm{~mL})$, mesitylene $(3.0 \mathrm{~mL})$, and the ampoule was immersed in an ultrasonic bath for $5 \mathrm{~min}$; following $0.4 \mathrm{~mL}$ of $6.0 \mathrm{~mol} \mathrm{~L}^{-1}$ aqueous acetic acid was added and the ampoule was immersed in an ultrasonic bath for $10 \mathrm{~min}$. Then the ampoule was flash frozen in a liquid nitrogen bath, evacuated to an internal pressure of 0 mbar and flame sealed, reducing the total length by ca. $10 \mathrm{~cm}$. Upon warming to room temperature, the ampoule was placed in an oven at $120^{\circ} \mathrm{C}$ and left undisturbed for 3 days, yielding a white solid. The ampoule was broken at the neck, and the white solid was isolated by centrifugation and washed with acetone $(3 \times 10 \mathrm{~mL})$, and soaking in dry acetone for $12 \mathrm{~h}$, and dried at $80{ }^{\circ} \mathrm{C}$ under vacuum for $12 \mathrm{~h}$ to yield COF-C6 as a white powder (65.7 mg, 93\% yield). 


\section{Scheme S5. Synthesis of COF-F4}
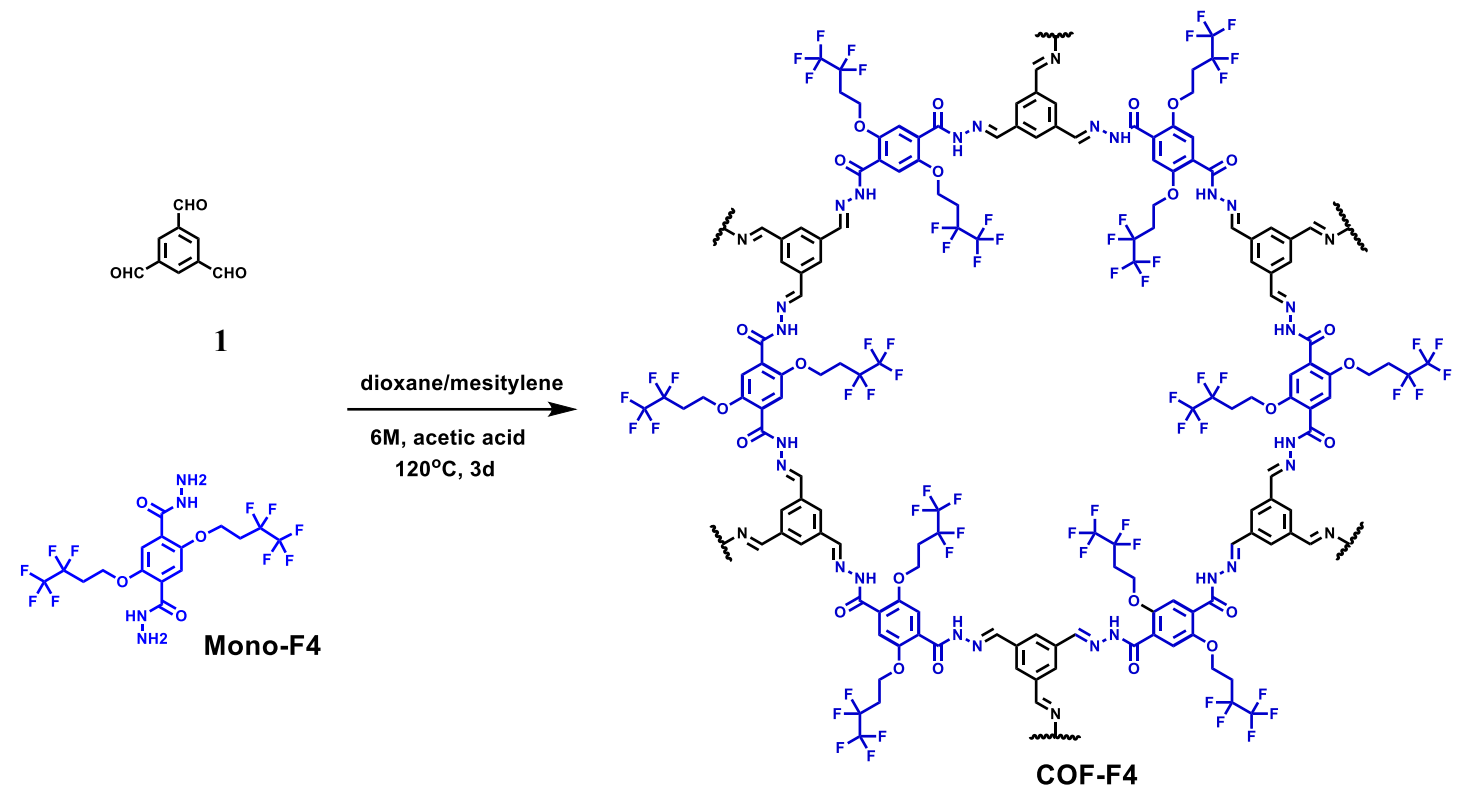

1,3,5-Trisformylbenzene 1 (16.2 mg, $0.10 \mathrm{mmol})$ and Mono-F4 (77.7 mg, $0.15 \mathrm{mmol})$ were weighed into a glass ampoule (volume of ca. $20 \mathrm{~mL}$, body length of $18 \mathrm{~cm}$, neck length of $9 \mathrm{~cm})$. To the mixture was added 1,4-dioxane $(1.0 \mathrm{~mL})$, mesitylene $(3.0 \mathrm{~mL})$, and the ampoule was immersed in an ultrasonic bath for $5 \mathrm{~min}$; following $0.4 \mathrm{~mL}$ of 6.0 mol L-1 aqueous acetic acid was added and the ampoule was immersed in an ultrasonic bath for $10 \mathrm{~min}$. Then the ampoule was flash frozen in a liquid nitrogen bath, evacuated to an internal pressure of 0 mbar and flame sealed, reducing the total length by ca. 10 $\mathrm{cm}$. Upon warming to room temperature, the ampoule was placed in an oven at $120{ }^{\circ} \mathrm{C}$ and left undisturbed for 3 days, yielding a white solid. The ampoule was broken at the neck, and the white solid was isolated by centrifugation and washed with acetone $(3 \times$ $10 \mathrm{~mL}$ ), and soaking in dry acetone for $12 \mathrm{~h}$, and dried at $80{ }^{\circ} \mathrm{C}$ under vacuum for $12 \mathrm{~h}$ to yield COF-F4 as white powder (67.0 $\mathrm{mg}, 76 \%$ yield). 


\section{Scheme S6. Synthesis of COF-F6}

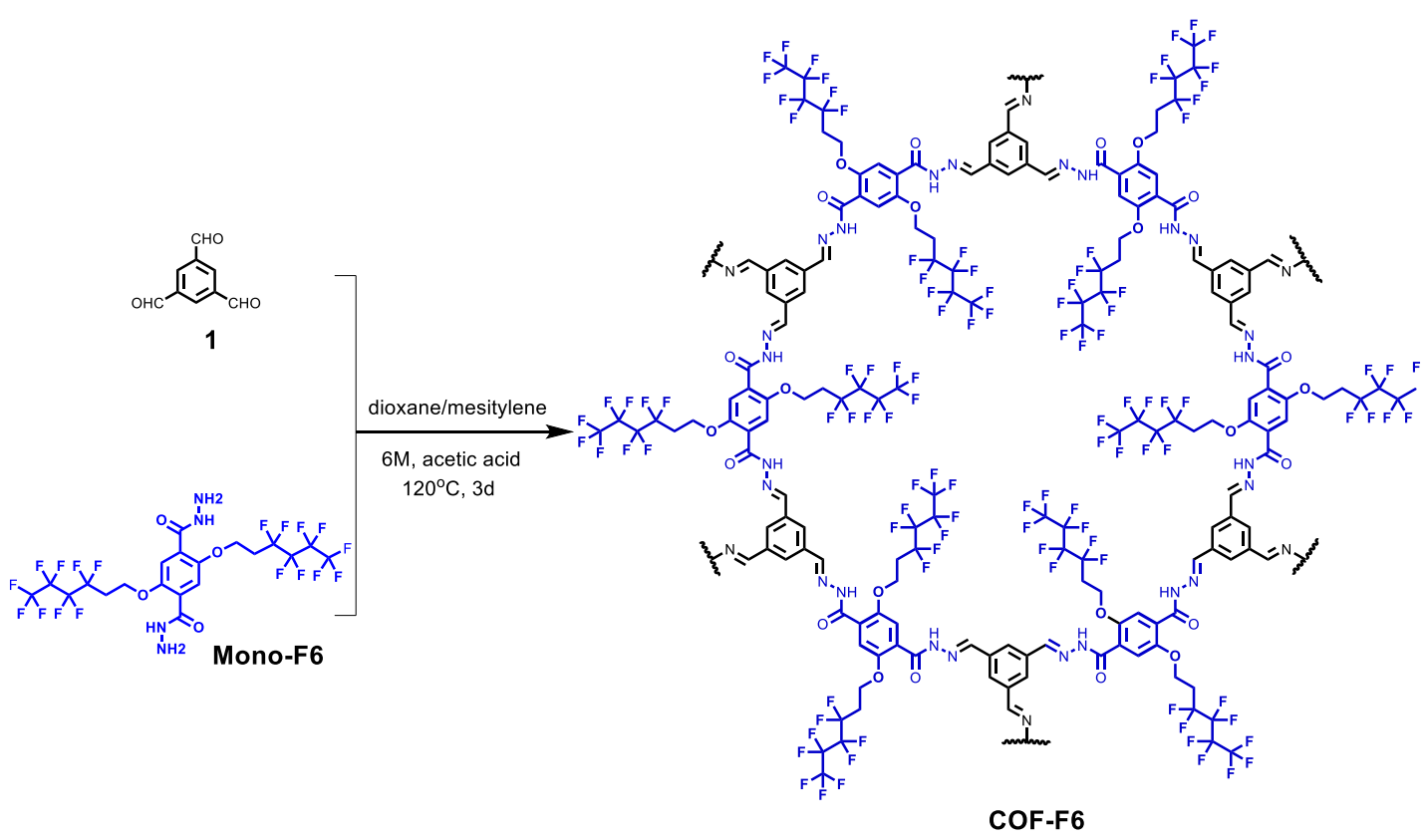

1,3,5-Trisformylbenzene 1 (16.2 $\mathrm{mg}, 0.10 \mathrm{mmol})$ and 2,5-bis((3,3,4,4,5,5,6,6,6nonafluorohexyl)oxy)terephthalohydrazide Mono-F6 (107.7 mg, $0.15 \mathrm{mmol}$ ) were weighed into a glass ampoule (volume of ca. $20 \mathrm{~mL}$, body length of $18 \mathrm{~cm}$, neck length of $9 \mathrm{~cm})$. To the mixture was added 1,4-dioxane $(1.0 \mathrm{~mL})$, mesitylene $(3.0 \mathrm{~mL})$, and the ampoule was immersed in an ultrasonic bath for $5 \mathrm{~min}$; following $0.4 \mathrm{~mL}$ of $6.0 \mathrm{~mol}$ $\mathrm{L}^{-1}$ aqueous acetic acid was added and the ampoule was immersed in an ultrasonic bath for $10 \mathrm{~min}$. Then the ampoule was flash frozen in a liquid nitrogen bath, evacuated to an internal pressure of 0 mbar and flame sealed, reducing the total length by $\mathrm{ca} .10 \mathrm{~cm}$. Upon warming to room temperature, the ampoule was placed in an oven at $120^{\circ} \mathrm{C}$ and left undisturbed for 3 days, yielding a white solid. The ampoule was broken at the neck, and the white solid was isolated by centrifugation and washed with acetone $(3 \times 10 \mathrm{~mL})$, and soaking in dry acetone for $12 \mathrm{~h}$, and dried at $80{ }^{\circ} \mathrm{C}$ under vacuum for $12 \mathrm{~h}$ to yield COF-F6 as white powder (100.0 mg, $81 \%$ yield). 


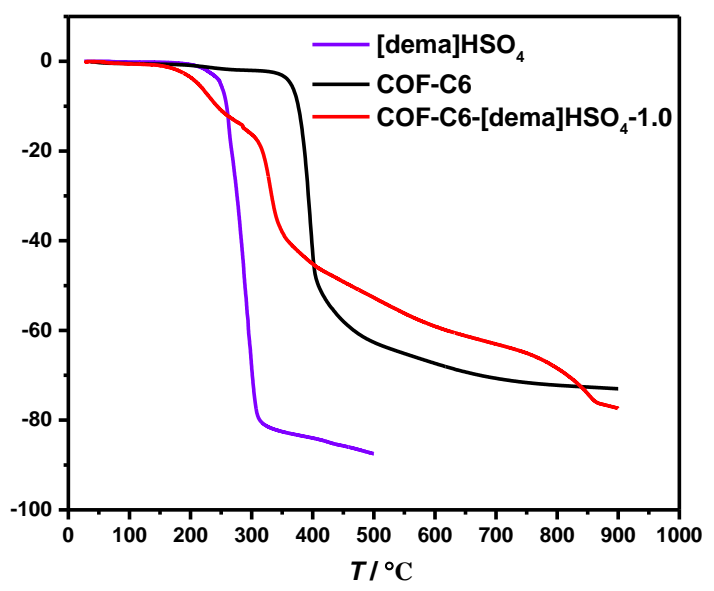

Figure S1. TGA profiles for C6-[dema]-HSO4-1.0

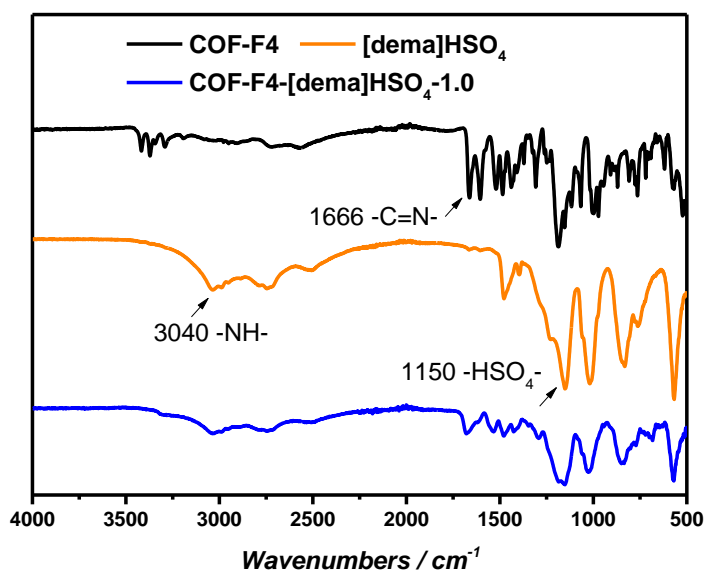

Figure S2. FT-IR spectra for F4-[dema]-HSO4-1.0

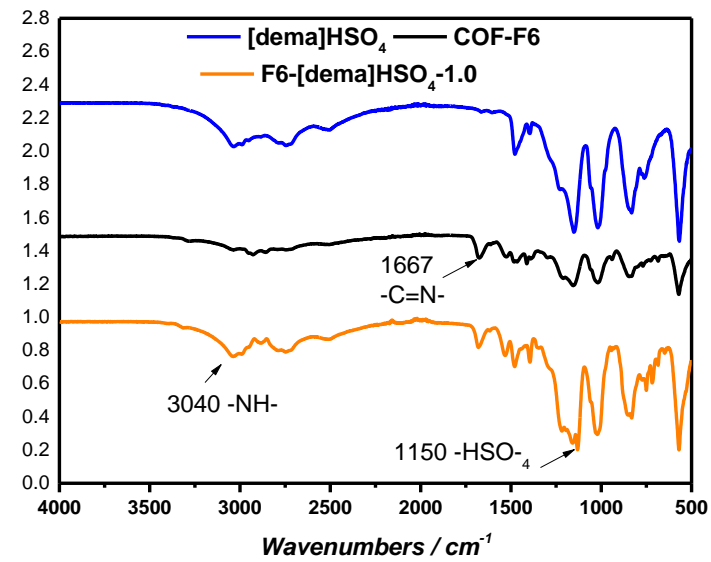

Figure S3. FT-IR spectra for F6-[dema]-HSO4-1.0 


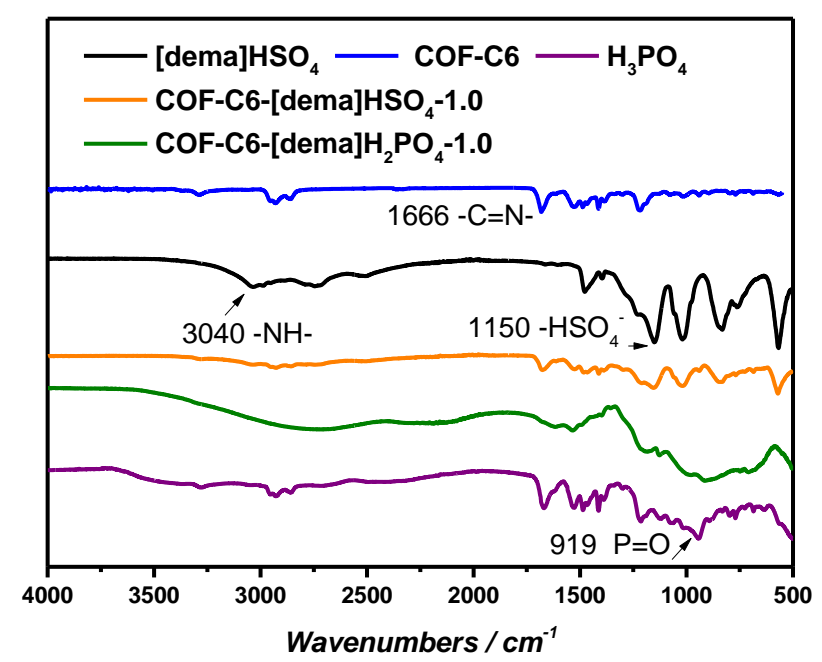

Figure S4. FT-IR spectra for C6-[dema]-IL-1.0

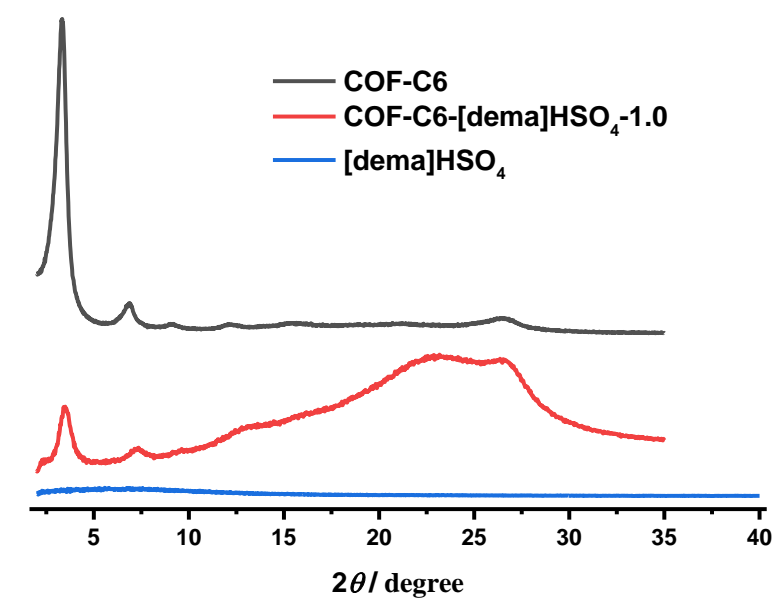

Figure S5. PXRD pattern for C6-[dema]-HSO4-1.0 


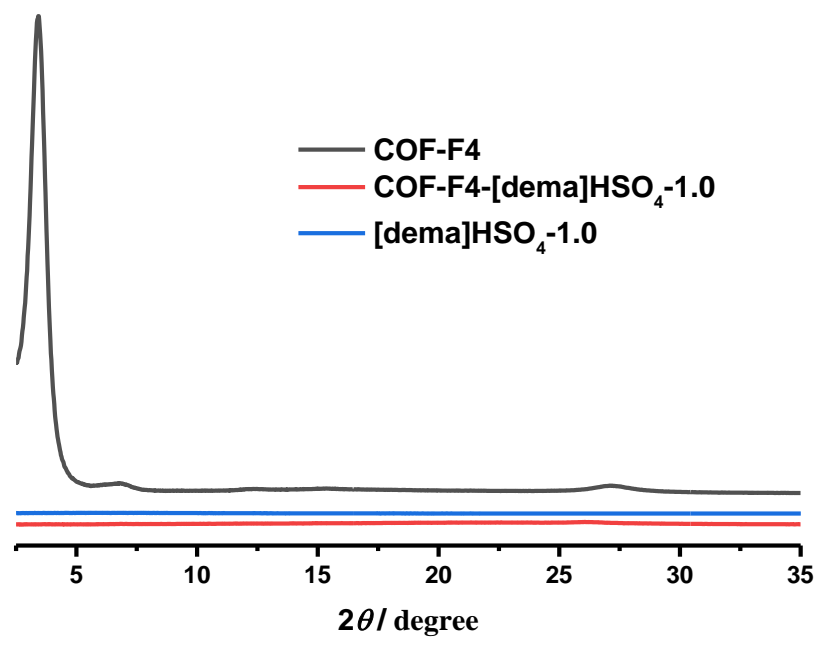

Figure S6. PXRD pattern for F4-[dema]-HSO4-1.0

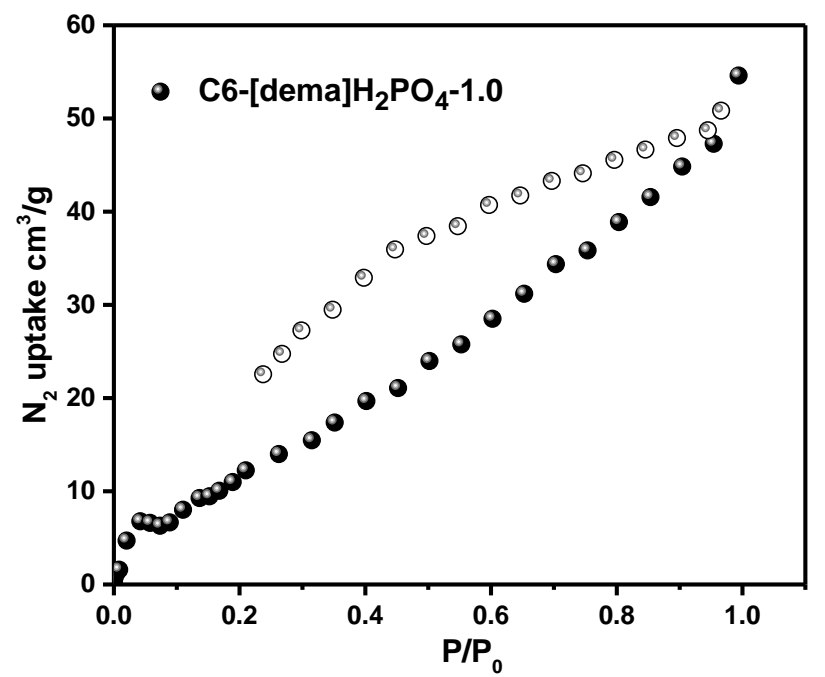

Figure S7. $\mathrm{N}_{2}$ sorption profile for $\mathbf{C 6}$-[dema] $\mathrm{H}_{2} \mathrm{PO}_{4}-\mathbf{1 . 0}$ 


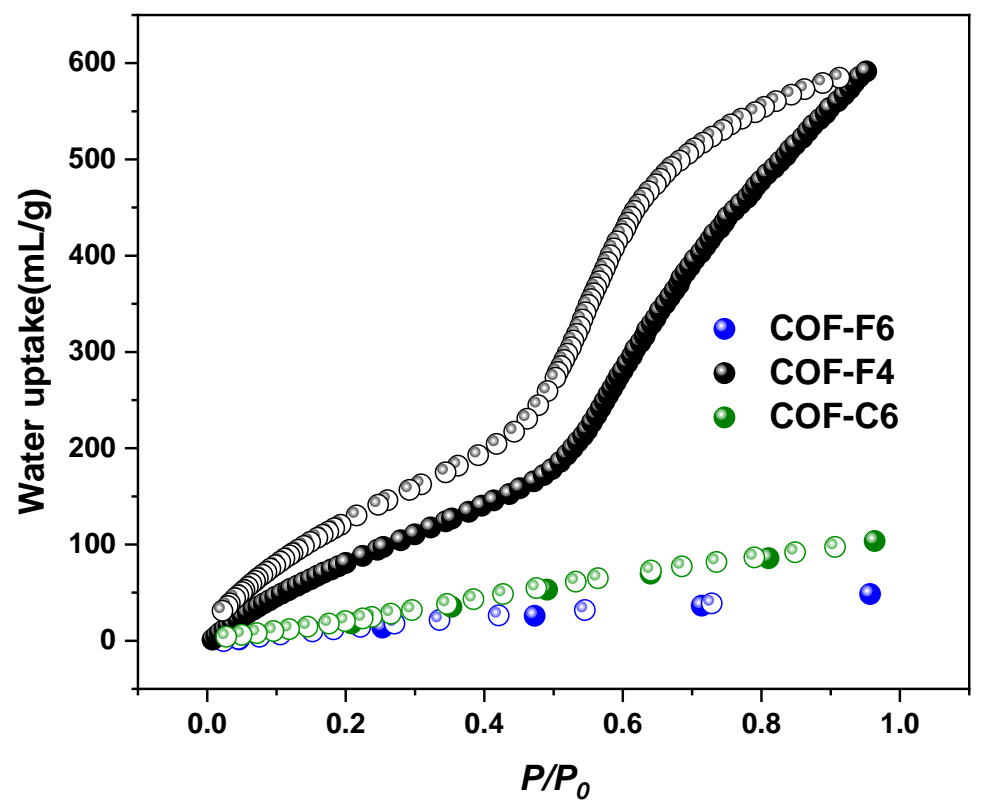

Figure S8. Water sorption curves for COF-C $\boldsymbol{x} / \mathbf{F} \boldsymbol{x}$

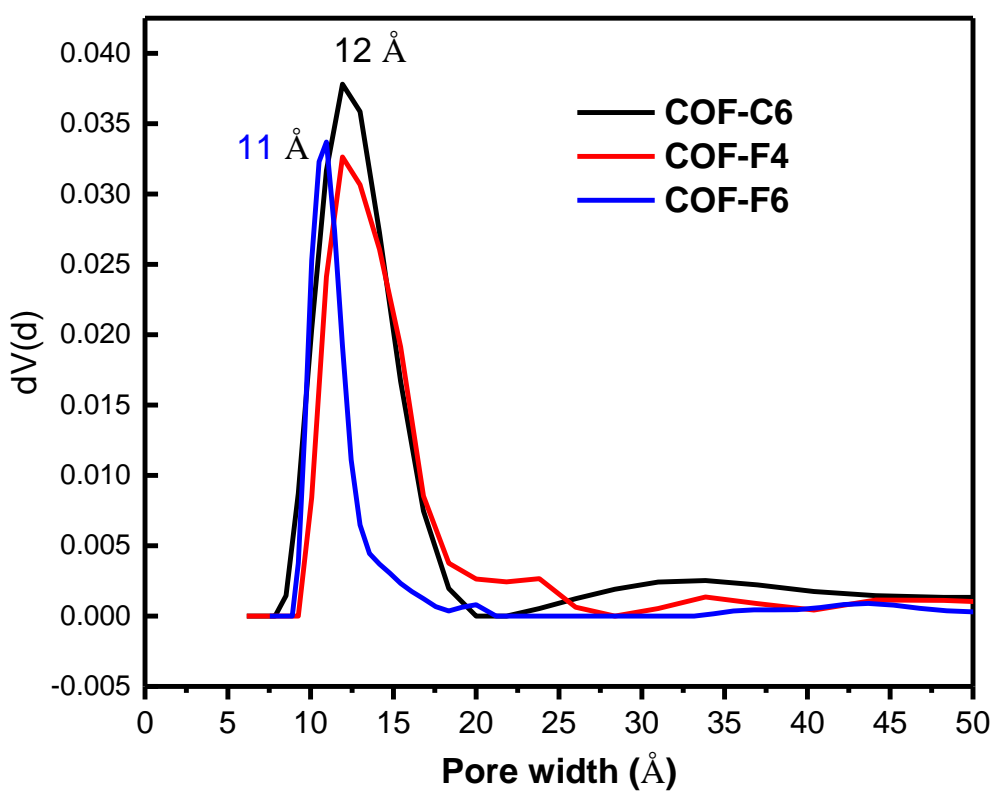

Figure S9. Pore size distribution for COF-Cx/Fx 


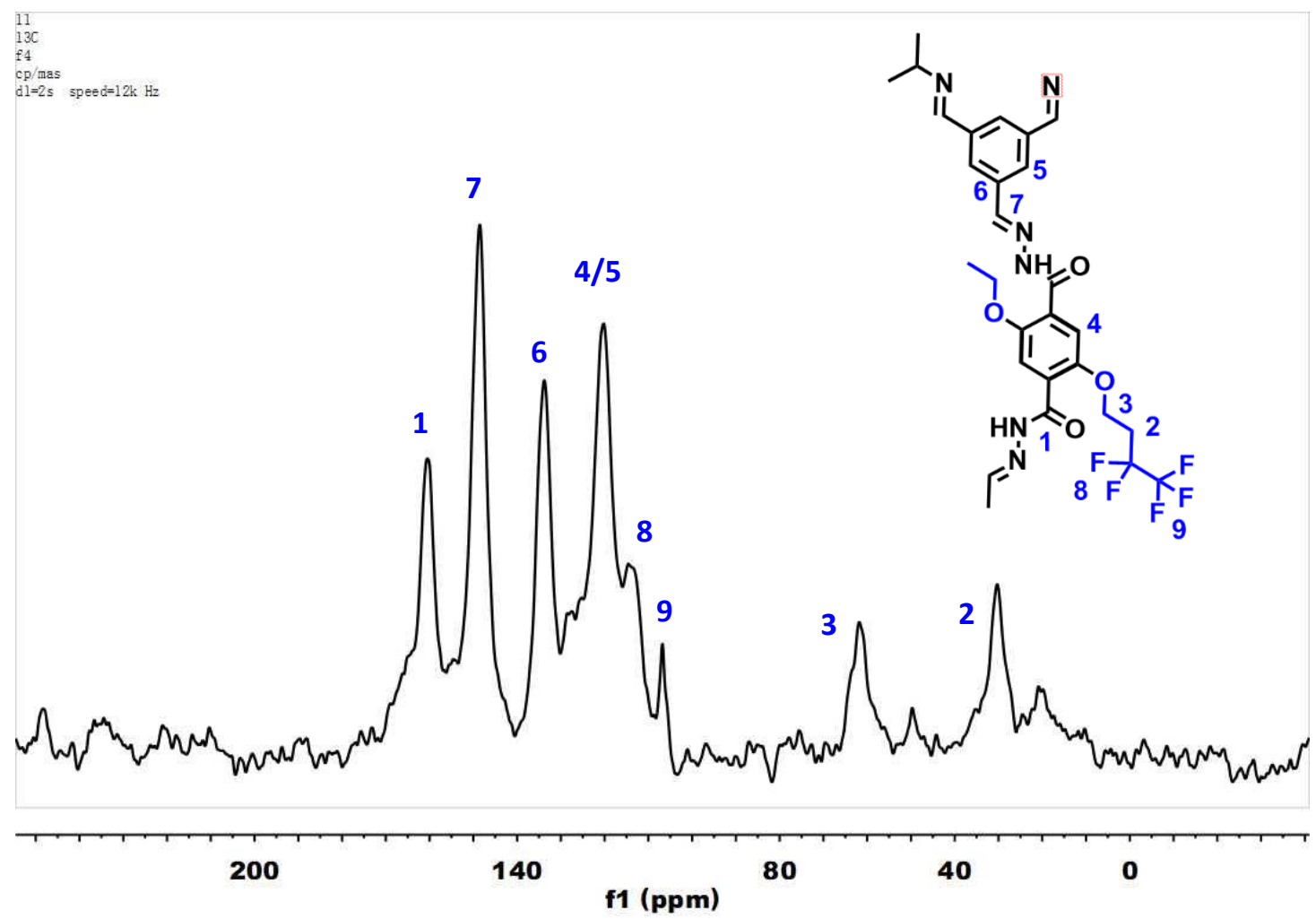

Figure $\mathbf{S 1 0}{ }^{13} \mathrm{C}$ CP-MAS spectra of COF-F4.

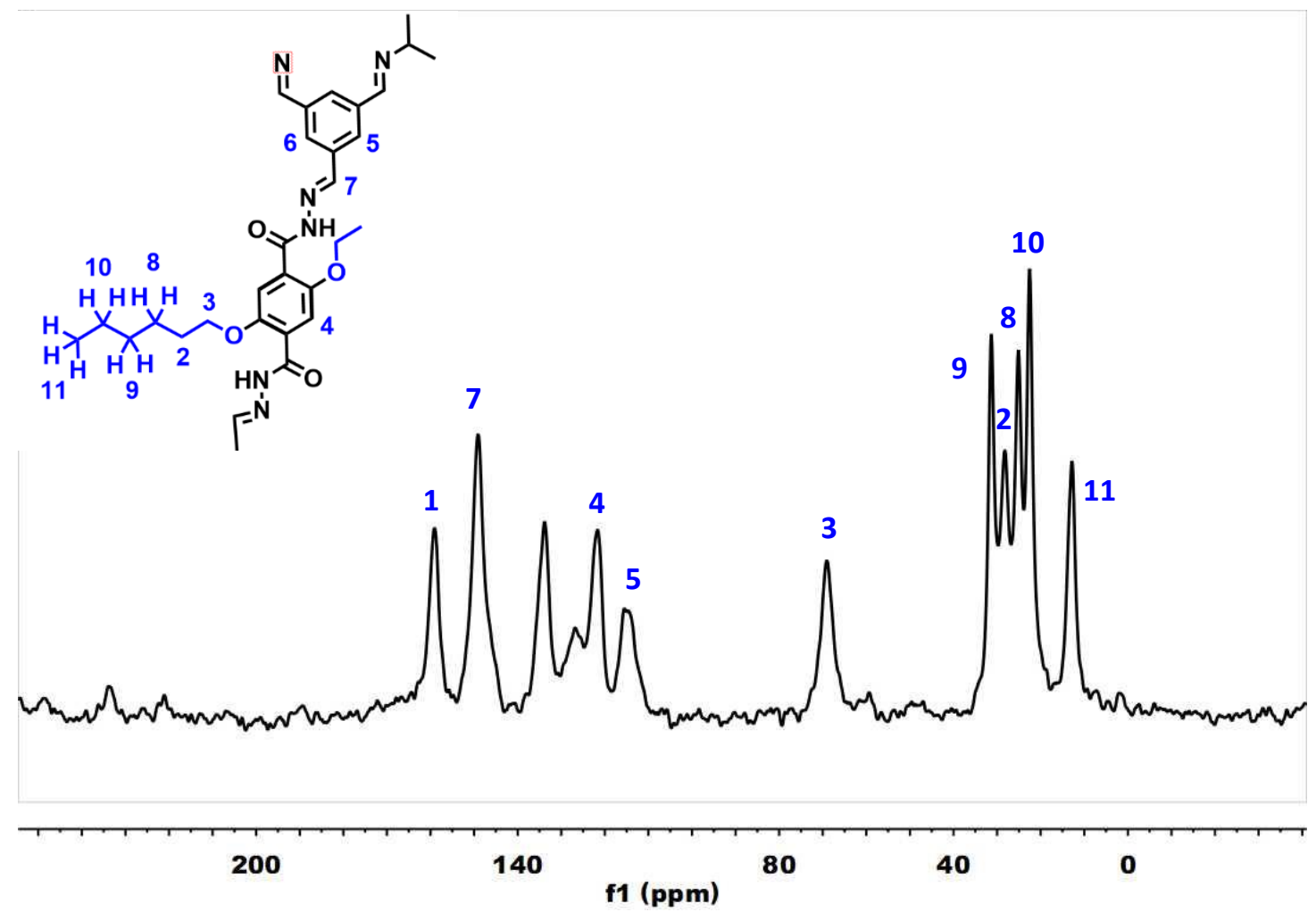

Figure S11 ${ }^{13} \mathrm{C}$ CP-MAS spectra of COF-C6. 


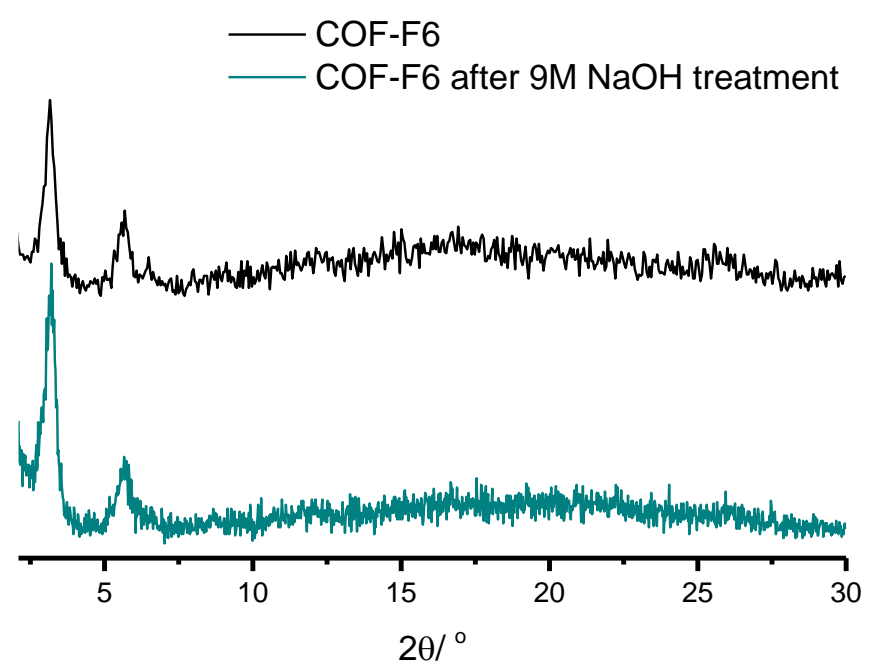

Figure S12 PXRD patterns of COF-F6 after base treatment.

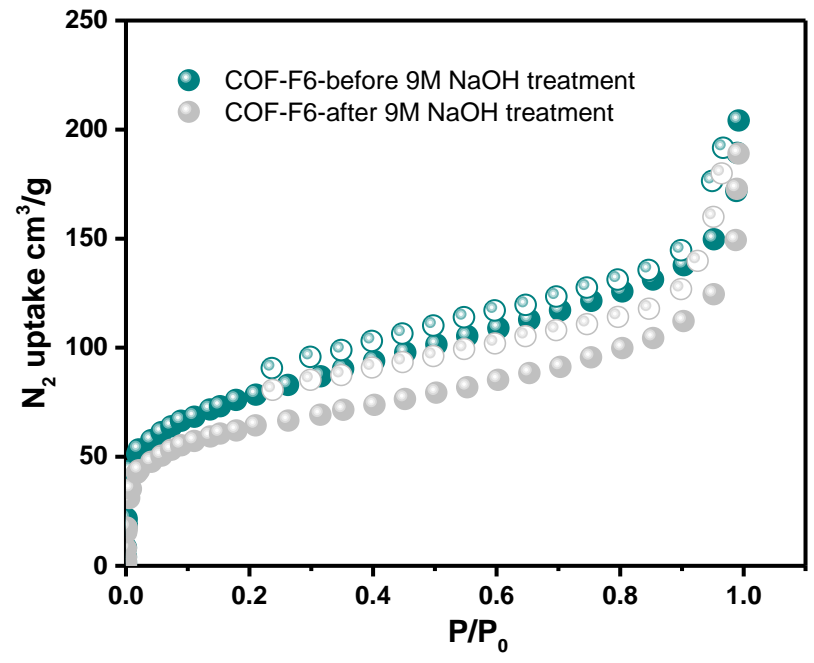

Figure $\mathrm{S} 13 \mathrm{~N}_{2}$ sorption of COF-F6 after base treatment.
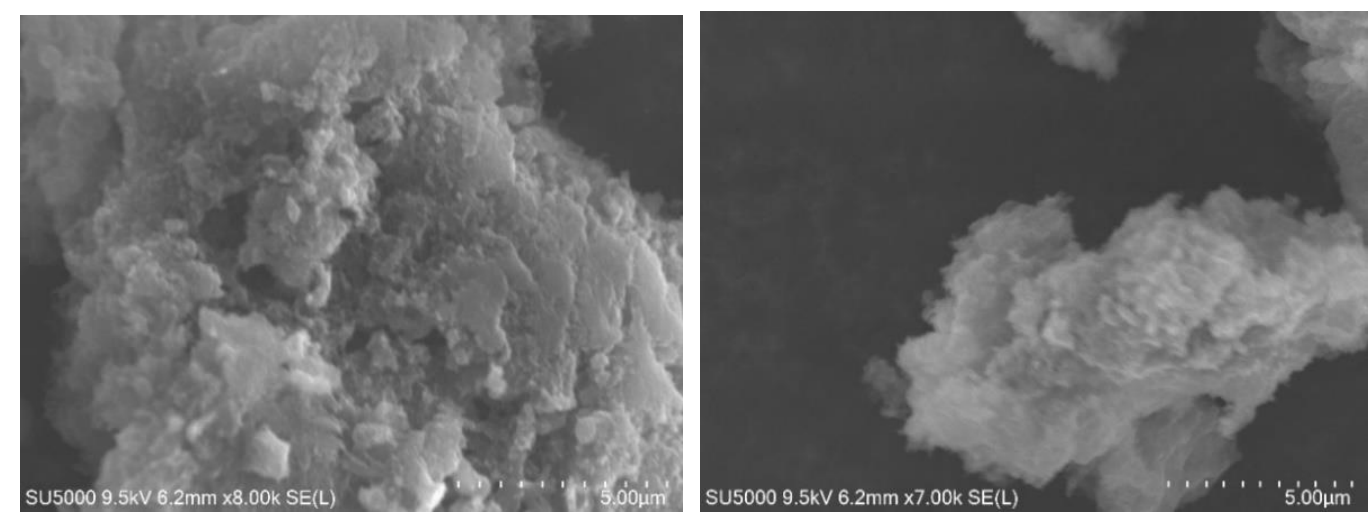

Figure S14. SEM images for COF-C6 


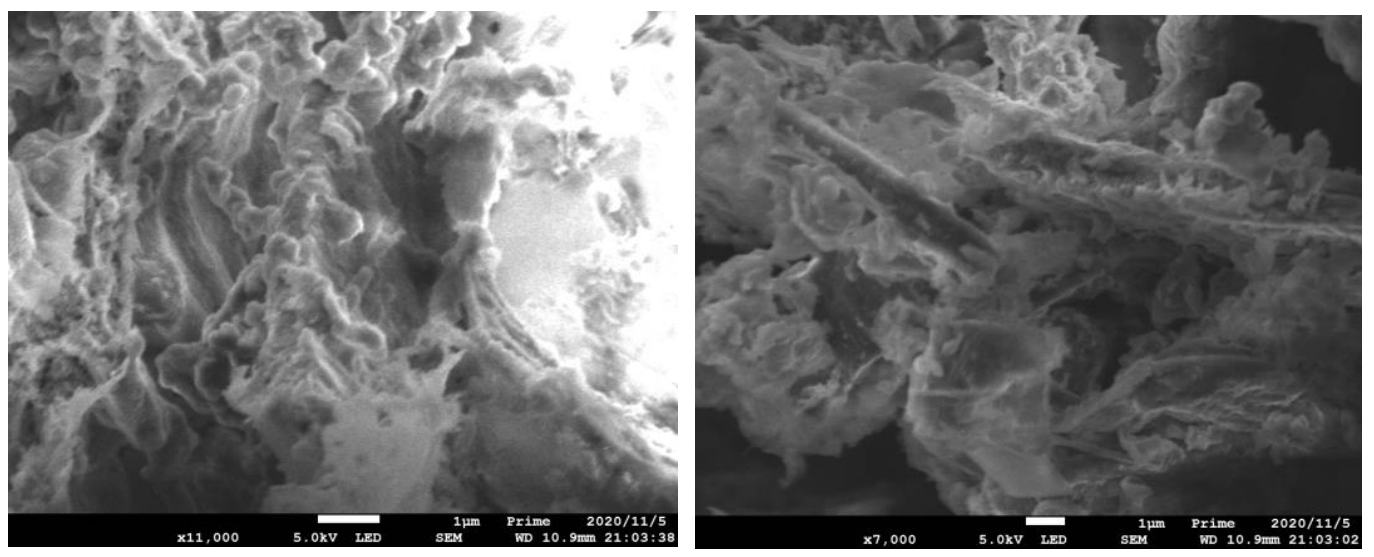

Figure S15. SEM images for COF-F4
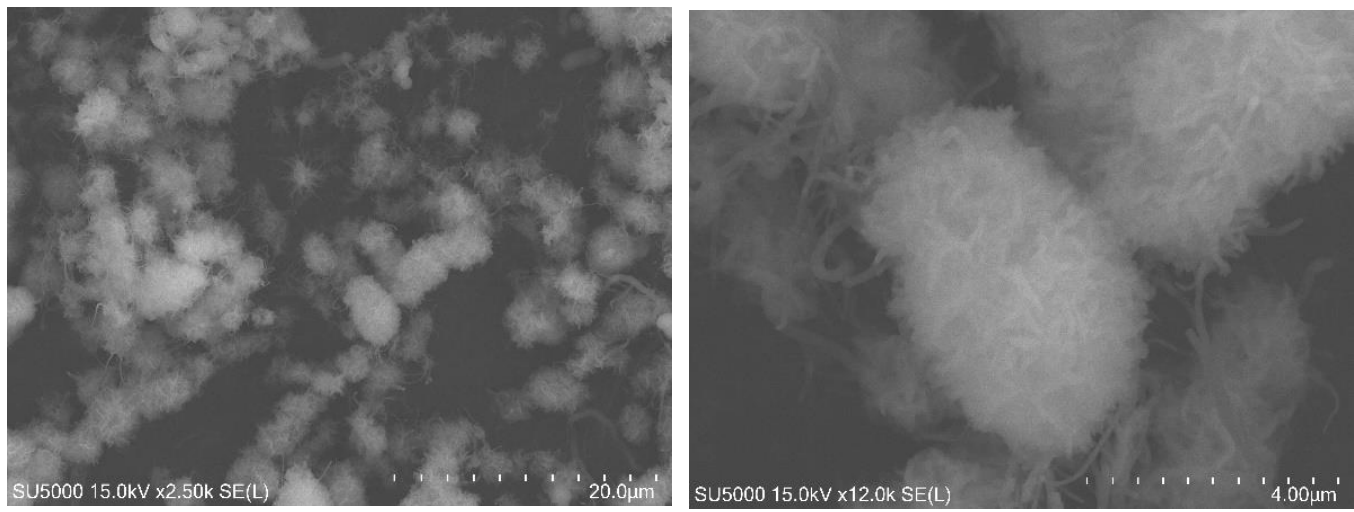

Figure S16. SEM images for COF-F6

(a)

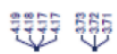

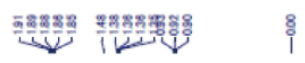
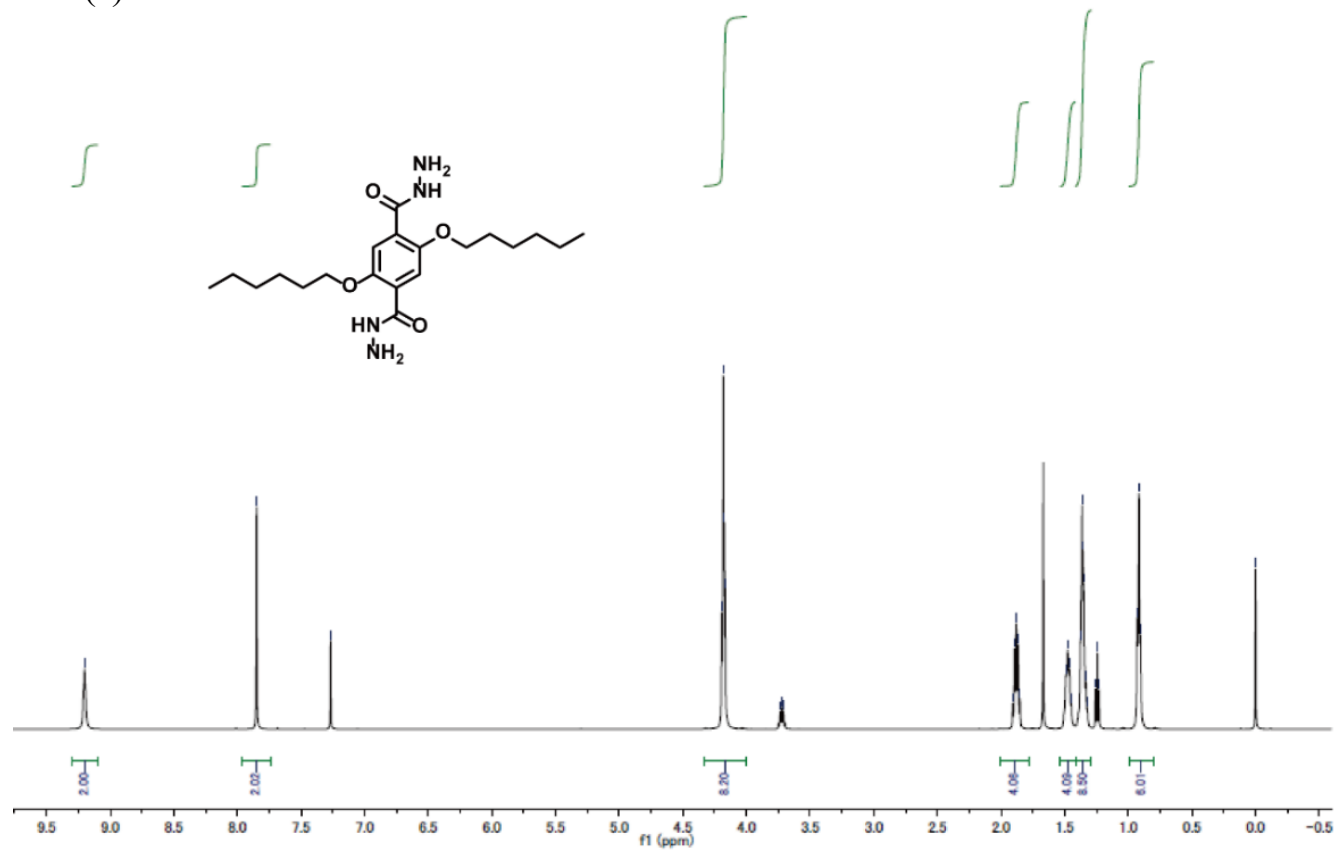
(b)

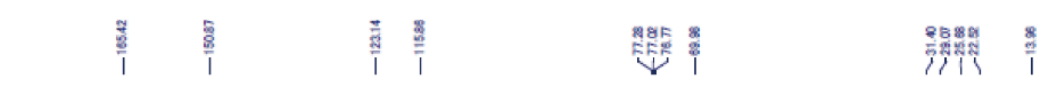
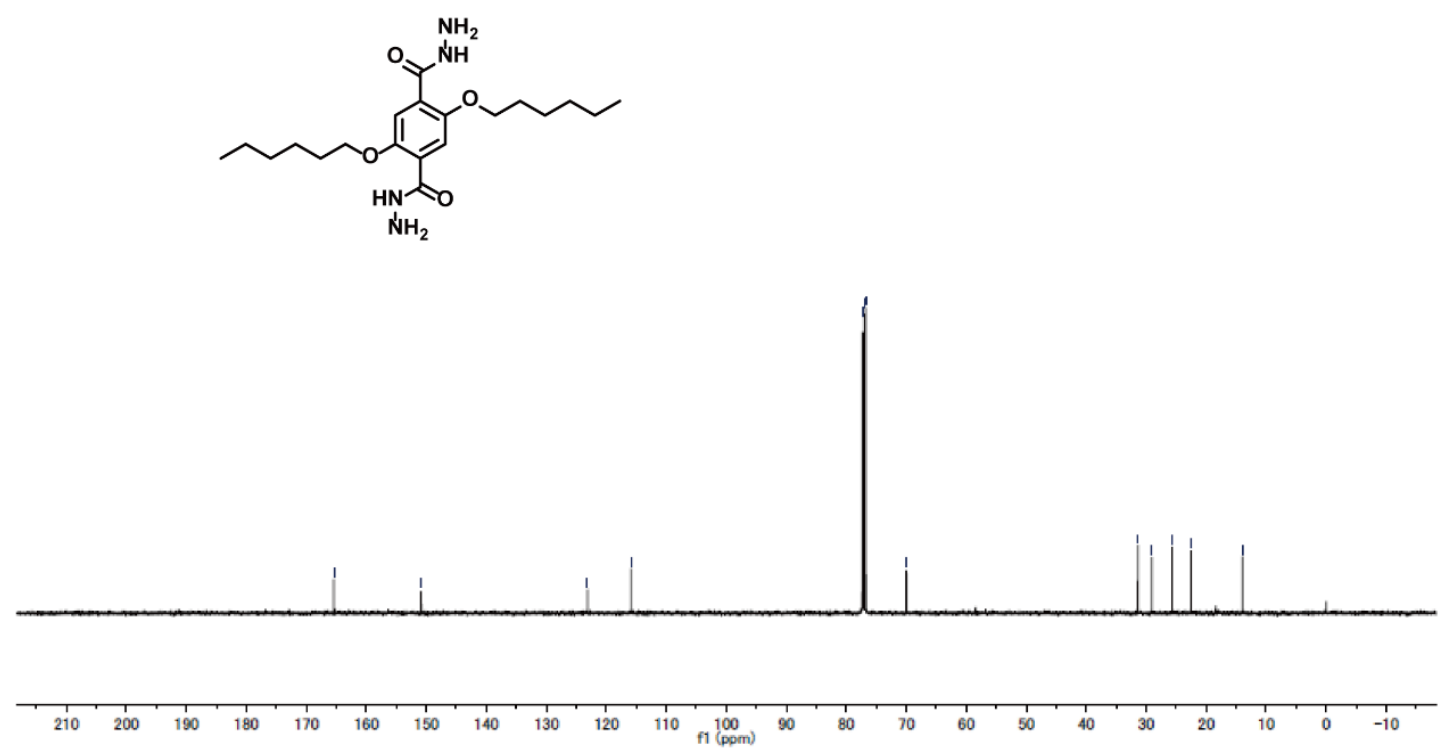

Figure S17. (a) ${ }^{1} \mathrm{H}$ NMR and (b) ${ }^{13} \mathrm{C}$ NMR spectra of Mono-C6.

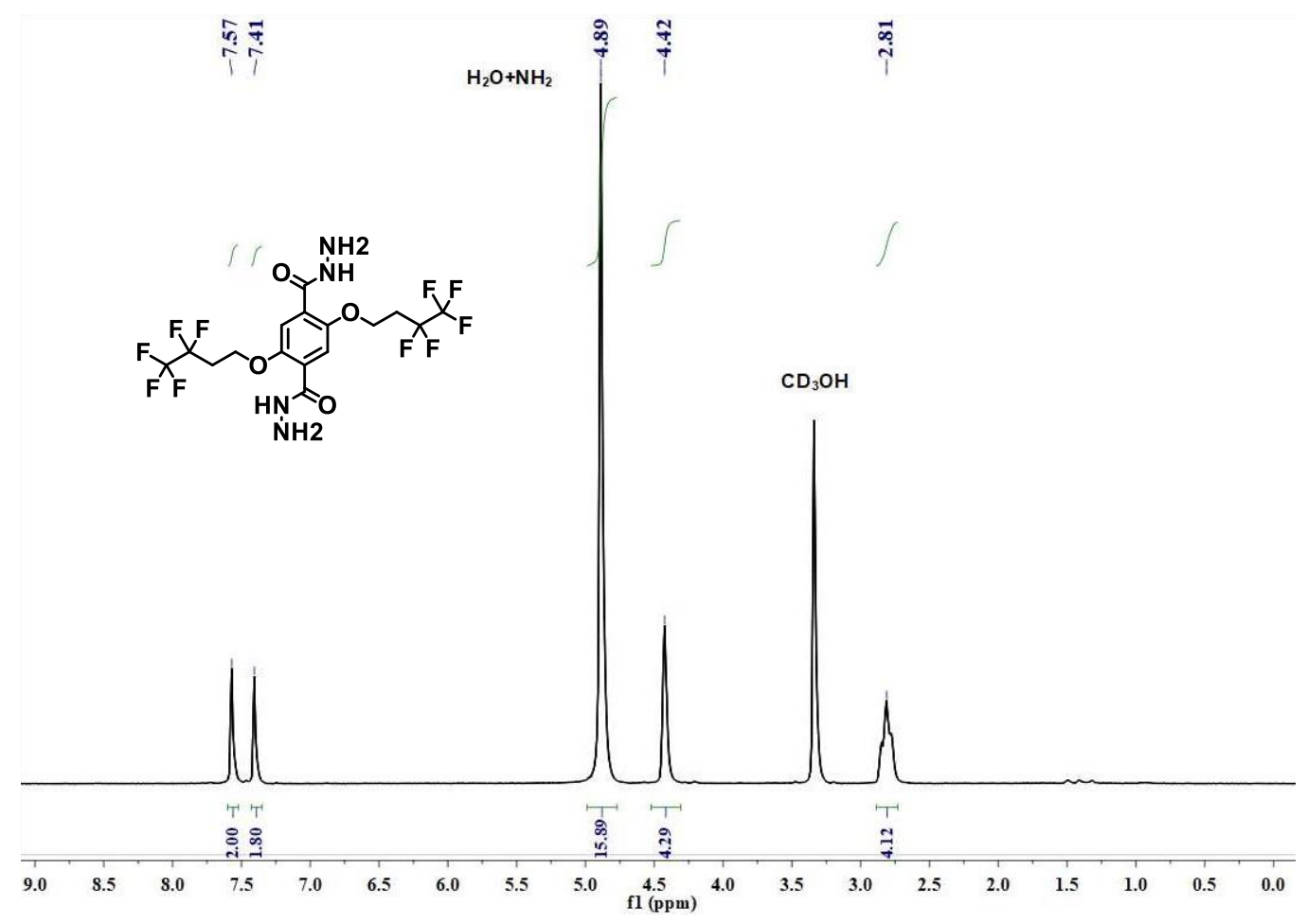

Figure S18. ${ }^{1} \mathrm{H}$ NMR spectrum of Mono-F4. 


\section{Structural Modeling of COFs}

Crystal structural modeling of COFs were generated by Material Studio, 2019 and optimized by the Density Functional Tight-Binding method (SCC-DFTB). Powder indexing and Pawley fitting were performed using Reflex module, Material Studio. Pore size distribution (PSD) were calculated from $\mathrm{N}_{2}$ sorption curves (experimental data).

Table S1. Fractional atomic coordinates for simulated COF-F4

\begin{tabular}{|c|c|c|c|}
\hline \multicolumn{4}{|c|}{ Space group: $P 3$} \\
\hline \multicolumn{4}{|c|}{$3 \mathrm{D}$ hexagonal; $\mathrm{a}=\mathrm{b}=29.63 \AA, \mathrm{c}=4.54 \AA ; \alpha=\beta=90^{\circ}, \gamma=120^{\circ}$} \\
\hline Atom & $\mathrm{x}$ & $\mathrm{y}$ & $\mathrm{Z}$ \\
\hline C1 & 0.2719 & -0.85472 & 0.75027 \\
\hline $\mathrm{C} 2$ & 0.29209 & -0.88804 & 0.81032 \\
\hline $\mathrm{C3}$ & 0.34382 & -0.87223 & 0.73669 \\
\hline C4 & 0.3752 & -0.8251 & 0.59012 \\
\hline $\mathrm{C} 5$ & 0.35406 & -0.79386 & 0.50348 \\
\hline C6 & 0.30258 & -0.80884 & 0.58819 \\
\hline 07 & 0.38535 & -0.74938 & 0.33007 \\
\hline $\mathrm{C} 8$ & 0.36683 & -0.71444 & 0.26128 \\
\hline $\mathrm{C9}$ & 0.41058 & -0.66629 & 0.1104 \\
\hline C10 & 0.39545 & -0.62445 & 0.04738 \\
\hline C11 & 0.44149 & -0.57493 & -0.08195 \\
\hline $\mathrm{C} 12$ & 0.4246 & -0.54147 & -0.1765 \\
\hline 014 & 0.2613 & -0.9361 & 0.95057 \\
\hline C15 & 0.25648 & -0.98109 & 0.80441 \\
\hline C16 & 0.19856 & -1.01996 & 0.75744 \\
\hline C17 & 0.18901 & -1.07197 & 0.62716 \\
\hline $\mathrm{C} 18$ & 0.13097 & -1.10901 & 0.56447 \\
\hline $\mathrm{C} 21$ & 0.43053 & -0.80877 & 0.53447 \\
\hline N22 & 0.46885 & -0.75826 & 0.57861 \\
\hline $\mathrm{O} 23$ & 0.44207 & -0.84123 & 0.45848 \\
\hline N24 & 0.5215 & -0.74373 & 0.54609 \\
\hline $\mathrm{C} 25$ & 0.5583 & -0.69558 & 0.56944 \\
\hline $\mathrm{C} 26$ & 0.61359 & -0.68124 & 0.55278 \\
\hline $\mathrm{C} 27$ & 0.62844 & -0.71945 & 0.55203 \\
\hline $\mathrm{C} 28$ & 0.21774 & -0.86841 & 0.83156 \\
\hline $\mathrm{O} 29$ & 0.19514 & -0.8517 & 0.6814 \\
\hline N30 & 0.19255 & -0.89928 & 1.06865 \\
\hline
\end{tabular}




\begin{tabular}{|l|l|l|l|} 
C31 & 0.11048 & -0.95923 & 1.26433 \\
\hline C32 & 0.05414 & -0.97972 & 1.28728 \\
\hline C33 & 0.03371 & -0.94603 & 1.28931 \\
\hline N34 & 0.13923 & -0.91773 & 1.11077 \\
\hline F35 & 0.37972 & -0.61118 & 0.30346 \\
\hline F36 & 0.35406 & -0.64418 & -0.14735 \\
\hline F37 & 0.46297 & -0.58708 & -0.31719 \\
\hline F38 & 0.47972 & -0.54967 & 0.12869 \\
\hline F44 & 0.20635 & -1.09607 & 0.82077 \\
\hline F45 & 0.21671 & -1.0625 & 0.36824 \\
\hline F46 & 0.10291 & -1.1233 & 0.8241 \\
\hline F47 & 0.11141 & -1.08532 & 0.38348 \\
\hline H53 & 0.35987 & -0.89663 & 0.79964 \\
\hline H54 & 0.28581 & -0.78503 & 0.52892 \\
\hline H55 & 0.35589 & -0.70215 & 0.46691 \\
\hline H56 & 0.33259 & -0.73386 & 0.112 \\
\hline H57 & 0.42047 & -0.67865 & -0.09938 \\
\hline H58 & 0.4457 & -0.64972 & 0.25334 \\
\hline H59 & 0.27418 & -0.99863 & 0.94614 \\
\hline H60 & 0.27665 & -0.97154 & 0.58719 \\
\hline H61 & 0.1829 & -1.00172 & 0.60611 \\
\hline H62 & 0.17774 & -1.0271 & 0.97063 \\
\hline H63 & 0.45923 & -0.73085 & 0.65527 \\
\hline H64 & 0.5485 & -0.66532 & 0.60667 \\
\hline H65 & 0.599 & -0.76039 & 0.55697 \\
\hline H66 & 0.21218 & -0.90996 & 1.21874 \\
\hline H67 & 0.12685 & -0.98199 & 1.35057 \\
\hline H68 & 0.05978 & -0.90423 & 1.28353 \\
\hline F69 & 1.15346 & 0.27811 & 0.42688 \\
\hline & & & \\
\hline
\end{tabular}

Table S2. Fractional atomic coordinates for simulated COF-F6

\begin{tabular}{|l|l|l|l|}
\hline \multicolumn{4}{|l|}{ Space group: $P 3$} \\
\hline \multicolumn{3}{|c|}{$\mathrm{xD}$ hexagonal; $\mathrm{a}=\mathrm{b}=29.79 \AA \mathrm{A}, \mathrm{c}=4.72 \AA ; \alpha=\beta=90^{\circ}, \gamma=120^{\circ}$} \\
\hline Atom & \multicolumn{1}{|c|}{$\mathrm{x}$} & $\mathrm{z}$ \\
\hline $\mathrm{C} 1$ & 0.27161 & -0.86703 & 0.71146 \\
\hline $\mathrm{C} 2$ & 0.29328 & -0.89779 & 0.78978 \\
\hline $\mathrm{C} 3$ & 0.34553 & -0.88056 & 0.73345 \\
\hline C4 & 0.37658 & -0.83385 & 0.59052 \\
\hline
\end{tabular}




\begin{tabular}{|c|c|c|c|}
\hline C5 & 0.35427 & -0.80496 & 0.48922 \\
\hline C6 & 0.30194 & -0.82179 & 0.55388 \\
\hline 07 & 0.38507 & -0.76109 & 0.3204 \\
\hline C8 & 0.36534 & -0.72781 & 0.24576 \\
\hline C9 & 0.4083 & -0.67929 & 0.10329 \\
\hline C10 & 0.39208 & -0.63843 & 0.04726 \\
\hline C11 & 0.43822 & -0.58738 & -0.06501 \\
\hline C12 & 0.42417 & -0.54424 & -0.10835 \\
\hline C13 & 0.47021 & -0.49316 & -0.21445 \\
\hline 014 & 0.26331 & -0.94511 & 0.92817 \\
\hline C15 & 0.25383 & -0.99021 & 0.7737 \\
\hline C16 & 0.19524 & -1.02553 & 0.73939 \\
\hline C17 & 0.18091 & -1.07902 & 0.61836 \\
\hline C18 & 0.12154 & -1.11335 & 0.57912 \\
\hline C19 & 0.10543 & -1.16871 & 0.47383 \\
\hline $\mathrm{C} 20$ & 0.04643 & -1.20342 & 0.44191 \\
\hline $\mathrm{C} 21$ & 0.43247 & -0.81589 & 0.55118 \\
\hline N22 & 0.46921 & -0.76488 & 0.5864 \\
\hline $\mathrm{O} 23$ & 0.44581 & -0.84748 & 0.49577 \\
\hline N24 & 0.52219 & -0.74862 & 0.56368 \\
\hline $\mathrm{C} 25$ & 0.55751 & -0.70015 & 0.58335 \\
\hline $\mathrm{C} 26$ & 0.61327 & -0.6835 & 0.57161 \\
\hline $\mathrm{C} 27$ & 0.6304 & -0.71969 & 0.57112 \\
\hline $\mathrm{C} 28$ & 0.21667 & -0.88252 & 0.77201 \\
\hline O29 & 0.19386 & -0.8692 & 0.6093 \\
\hline N30 & 0.19107 & -0.91094 & 1.00448 \\
\hline C31 & 0.10862 & -0.96618 & 1.19979 \\
\hline C32 & 0.05321 & -0.98319 & 1.22104 \\
\hline C33 & 0.03623 & -0.94697 & 1.22287 \\
\hline N34 & 0.13847 & -0.92705 & 1.04193 \\
\hline F35 & 0.37396 & -0.62868 & 0.29427 \\
\hline F36 & 0.35211 & -0.65815 & -0.14703 \\
\hline F37 & 0.45427 & -0.59768 & -0.31797 \\
\hline F38 & 0.47902 & -0.56997 & 0.12315 \\
\hline F39 & 0.38412 & -0.56064 & -0.30032 \\
\hline F40 & 0.40758 & -0.53404 & 0.14304 \\
\hline F41 & 0.4901 & -0.50089 & -0.46022 \\
\hline F42 & 0.50914 & -0.47229 & -0.01483 \\
\hline F43 & 0.45388 & -0.45794 & -0.26684 \\
\hline
\end{tabular}




\begin{tabular}{|l|l|l|l|}
\hline F44 & 0.19875 & -1.103 & 0.79972 \\
\hline F45 & 0.20534 & -1.0722 & 0.36126 \\
\hline F46 & 0.09712 & -1.11691 & 0.83253 \\
\hline F47 & 0.10535 & -1.08946 & 0.38694 \\
\hline F48 & 0.12828 & -1.16558 & 0.21607 \\
\hline F49 & 0.12243 & -1.19265 & 0.66068 \\
\hline F50 & 0.03547 & -1.2503 & 0.32459 \\
\hline F51 & 0.02631 & -1.18052 & 0.26876 \\
\hline F52 & 0.02258 & -1.21296 & 0.70207 \\
\hline H53 & 0.3621 & -0.90357 & 0.8064 \\
\hline H54 & 0.28413 & -0.79995 & 0.48214 \\
\hline H55 & 0.35291 & -0.71611 & 0.43944 \\
\hline H56 & 0.33208 & -0.74812 & 0.09869 \\
\hline H57 & 0.41879 & -0.69057 & -0.09863 \\
\hline H58 & 0.44288 & -0.66271 & 0.24258 \\
\hline H59 & 0.27022 & -1.01044 & 0.8955 \\
\hline H60 & 0.27237 & -0.98073 & 0.56169 \\
\hline H61 & 0.18065 & -1.00593 & 0.59817 \\
\hline H62 & 0.17651 & -1.03049 & 0.9483 \\
\hline H63 & 0.45802 & -0.73814 & 0.64593 \\
\hline H64 & 0.54616 & -0.67111 & 0.61173 \\
\hline H65 & 0.60246 & -0.76077 & 0.57463 \\
\hline H66 & 0.21009 & -0.92036 & 1.15754 \\
\hline H67 & 0.12324 & -0.98937 & 1.29471 \\
\hline H68 & 0.06425 & -0.9059 & 1.21748 \\
\hline & & \\
\hline
\end{tabular}

Table S3. Fractional atomic coordinates for simulated COF-C6

\begin{tabular}{|l|l|l|l|}
\hline \multicolumn{4}{|l|}{ Space group: $P 3$} \\
\hline \multicolumn{3}{|c|}{$\mathrm{x}$ 3 hexagonal; $\mathrm{a}=\mathrm{b}=29.40 \AA \mathrm{A}, \mathrm{c}=3.99 \AA ; \alpha=\beta=90^{\circ}, \gamma=120^{\circ}$} \\
\hline Atom & \multicolumn{1}{|c|}{$\mathrm{y}$} & \multicolumn{1}{c|}{$\mathrm{z}$} \\
\hline $\mathrm{C} 1$ & 0.26937 & -0.84963 & 0.77568 \\
\hline $\mathrm{C} 2$ & 0.2863 & -0.88696 & 0.76396 \\
\hline $\mathrm{C} 3$ & 0.3386 & -0.8703 & 0.67775 \\
\hline $\mathrm{C} 4$ & 0.373 & -0.81831 & 0.58048 \\
\hline $\mathrm{C} 5$ & 0.35437 & -0.78269 & 0.5498 \\
\hline $\mathrm{C} 6$ & 0.30268 & -0.79872 & 0.65359 \\
\hline O7 & 0.38854 & -0.73244 & 0.41534 \\
\hline $\mathrm{C} 8$ & 0.36922 & -0.6972 & 0.35679 \\
\hline
\end{tabular}




\begin{tabular}{|c|c|c|c|}
\hline C9 & 0.41402 & -0.64535 & 0.22445 \\
\hline C10 & 0.39578 & -0.60552 & 0.16141 \\
\hline C11 & 0.44147 & -0.55277 & 0.04309 \\
\hline C12 & 0.4232 & -0.51312 & -0.02569 \\
\hline C13 & 0.46867 & -0.46053 & -0.1426 \\
\hline 014 & 0.25136 & -0.93945 & 0.85433 \\
\hline C15 & 0.25379 & -0.98076 & 0.67947 \\
\hline C16 & 0.19789 & -1.02268 & 0.58338 \\
\hline C17 & 0.19359 & -1.07497 & 0.48204 \\
\hline C18 & 0.13622 & -1.11607 & 0.40497 \\
\hline C19 & 0.13072 & -1.16863 & 0.29711 \\
\hline $\mathrm{C} 20$ & 0.0735 & -1.20936 & 0.22397 \\
\hline C21 & 0.42881 & -0.80172 & 0.51938 \\
\hline N22 & 0.46786 & -0.75416 & 0.63174 \\
\hline $\mathrm{O} 23$ & 0.43999 & -0.83165 & 0.37807 \\
\hline N24 & 0.52072 & -0.74026 & 0.59715 \\
\hline C25 & 0.55833 & -0.69323 & 0.66423 \\
\hline C26 & 0.6136 & -0.68004 & 0.64635 \\
\hline C27 & 0.62723 & -0.71949 & 0.64598 \\
\hline C28 & 0.21598 & -0.86331 & 0.89366 \\
\hline $\mathrm{O} 29$ & 0.19475 & -0.84035 & 0.77511 \\
\hline N30 & 0.19036 & -0.90075 & 1.13352 \\
\hline C31 & 0.11086 & -0.95789 & 1.4042 \\
\hline C32 & 0.0545 & -0.97894 & 1.44636 \\
\hline C33 & 0.03337 & -0.94554 & 1.44968 \\
\hline N34 & 0.13758 & -0.9182 & 1.20782 \\
\hline F35 & 0.37921 & -0.5994 & 0.39556 \\
\hline F36 & 0.36408 & -0.62131 & -0.02974 \\
\hline F37 & 0.4585 & -0.55897 & -0.18873 \\
\hline F38 & 0.47277 & -0.53672 & 0.23689 \\
\hline F39 & 0.40612 & -0.5066 & 0.2049 \\
\hline F40 & 0.39215 & -0.52882 & -0.22092 \\
\hline F41 & 0.48797 & -0.46607 & -0.36503 \\
\hline F42 & 0.49832 & -0.44199 & 0.05937 \\
\hline $\mathrm{F} 43$ & 0.45373 & -0.43374 & -0.21037 \\
\hline F44 & 0.20832 & -1.08955 & 0.6878 \\
\hline F45 & 0.21841 & -1.06876 & 0.25828 \\
\hline F46 & 0.12119 & -1.10108 & 0.20305 \\
\hline F47 & 0.1117 & -1.12236 & 0.63072 \\
\hline
\end{tabular}




\begin{tabular}{|l|l|l|l|}
\hline F48 & 0.14563 & -1.18402 & 0.49714 \\
\hline F49 & 0.15457 & -1.16277 & 0.06899 \\
\hline F50 & 0.07114 & -1.24577 & 0.12467 \\
\hline F51 & 0.05671 & -1.19412 & 0.03557 \\
\hline F52 & 0.04964 & -1.21927 & 0.45621 \\
\hline H53 & 0.35312 & -0.89756 & 0.69729 \\
\hline H54 & 0.28789 & -0.7717 & 0.64475 \\
\hline H55 & 0.35477 & -0.68929 & 0.59381 \\
\hline H56 & 0.33673 & -0.71446 & 0.17106 \\
\hline H57 & 0.42929 & -0.65282 & -0.01111 \\
\hline H58 & 0.44656 & -0.6291 & 0.4099 \\
\hline H59 & 0.27113 & -0.99788 & 0.84995 \\
\hline H60 & 0.27807 & -0.96682 & 0.44822 \\
\hline H61 & 0.18495 & -1.00771 & 0.37058 \\
\hline H62 & 0.17117 & -1.02944 & 0.79787 \\
\hline H63 & 0.45848 & -0.72926 & 0.76419 \\
\hline H64 & 0.54923 & -0.66307 & 0.7354 \\
\hline H65 & 0.59681 & -0.76047 & 0.65194 \\
\hline H66 & 0.20965 & -0.91698 & 1.26239 \\
\hline H67 & 0.12856 & -0.97917 & 1.51003 \\
\hline H68 & 0.05921 & -0.90334 & 1.43885 \\
\hline & & & \\
\hline
\end{tabular}

(a) $-20^{\circ} \mathrm{C}$

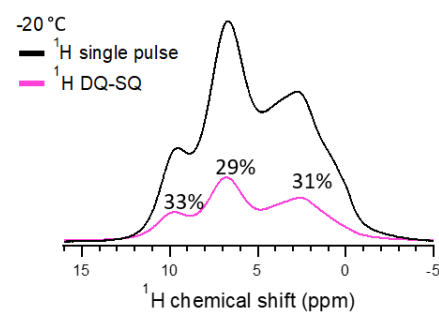

(b)

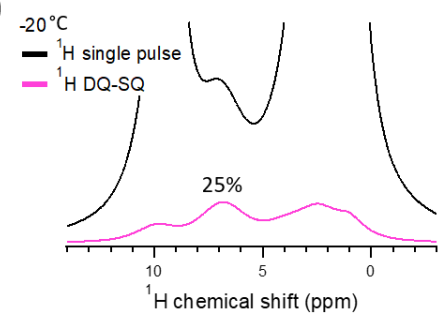

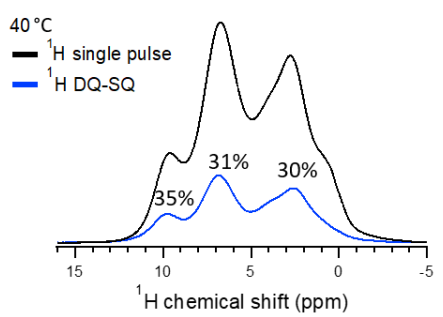
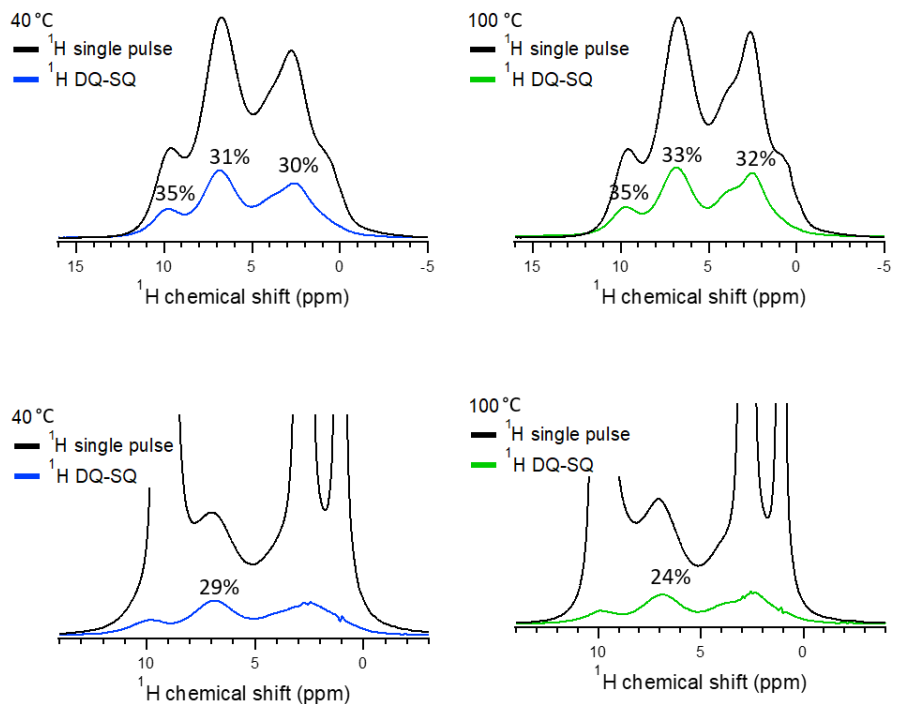

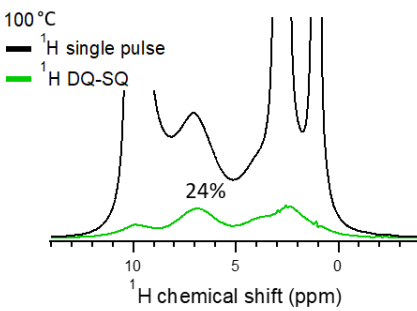

Figure S19. 1D ${ }^{1} \mathrm{H}$ single pulse and DQ-SQ of (a) COF-F6, (b) F6-[dema]HSO 4 -1.0,

(IL is short for [dema]HSO 4 in this ESI) measured at $-20,40$, and $100{ }^{\circ} \mathrm{C}$. The number denotes the ${ }^{1} \mathrm{H}$ DQ filtering efficiency comparing to the ${ }^{1} \mathrm{H}$ signal intensity of the single 
pulse.

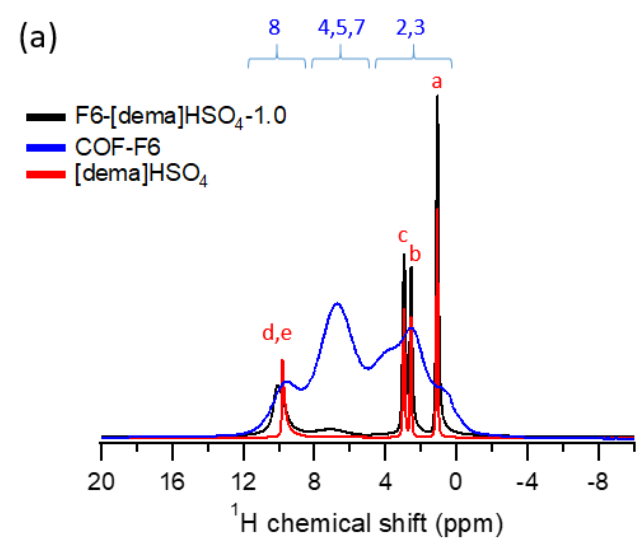

(c)

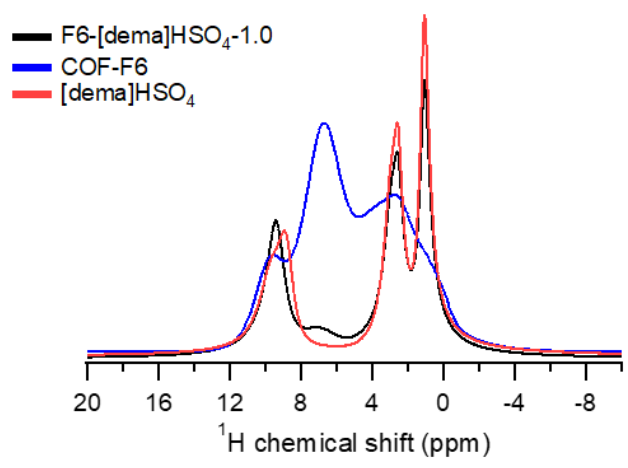

(b)

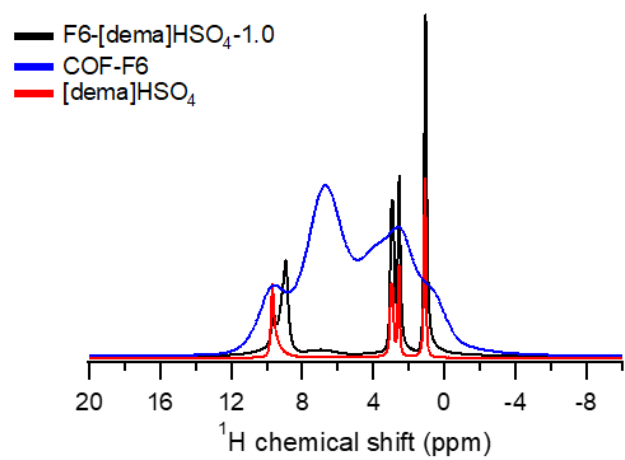

(d)

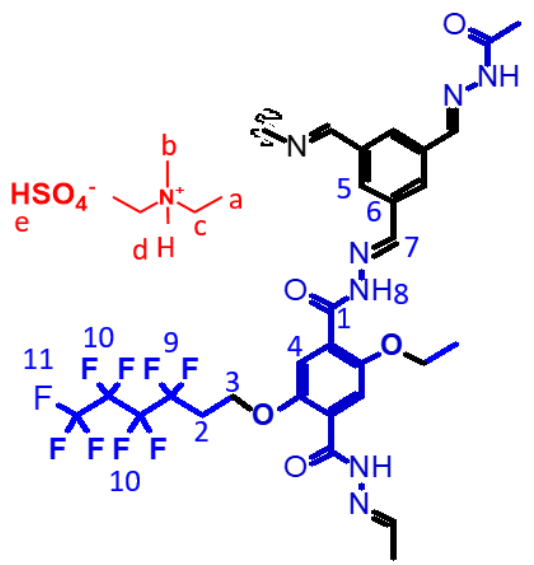

Figure S20. (a-c) ${ }^{1} \mathrm{H}$ single pulse spectra of F6-[dema]HSO4-1.0, F6, and IL ([dema]HSO 4 ) measured at -20 (a), 40 (b), and $100{ }^{\circ} \mathrm{C}$ (c) with the ${ }^{1} \mathrm{H}$ assignment of the structural formula of F6-[dema] $\mathrm{HSO}_{4}$.

*Note: The comparison of IL and F6-IL spectra measured at low and high temperature of -20 and $100{ }^{\circ} \mathrm{C}$ shows identical ${ }^{1} \mathrm{H}$ chemical shifts of dema (a,b,c peak), which means that the chemical environment of IL in COF-F6 system would be the same as that of pristine IL. The small differences of peak $\mathbf{d}$ and $\mathbf{e}$ would come from hydrogen bonding to COF-F6. 

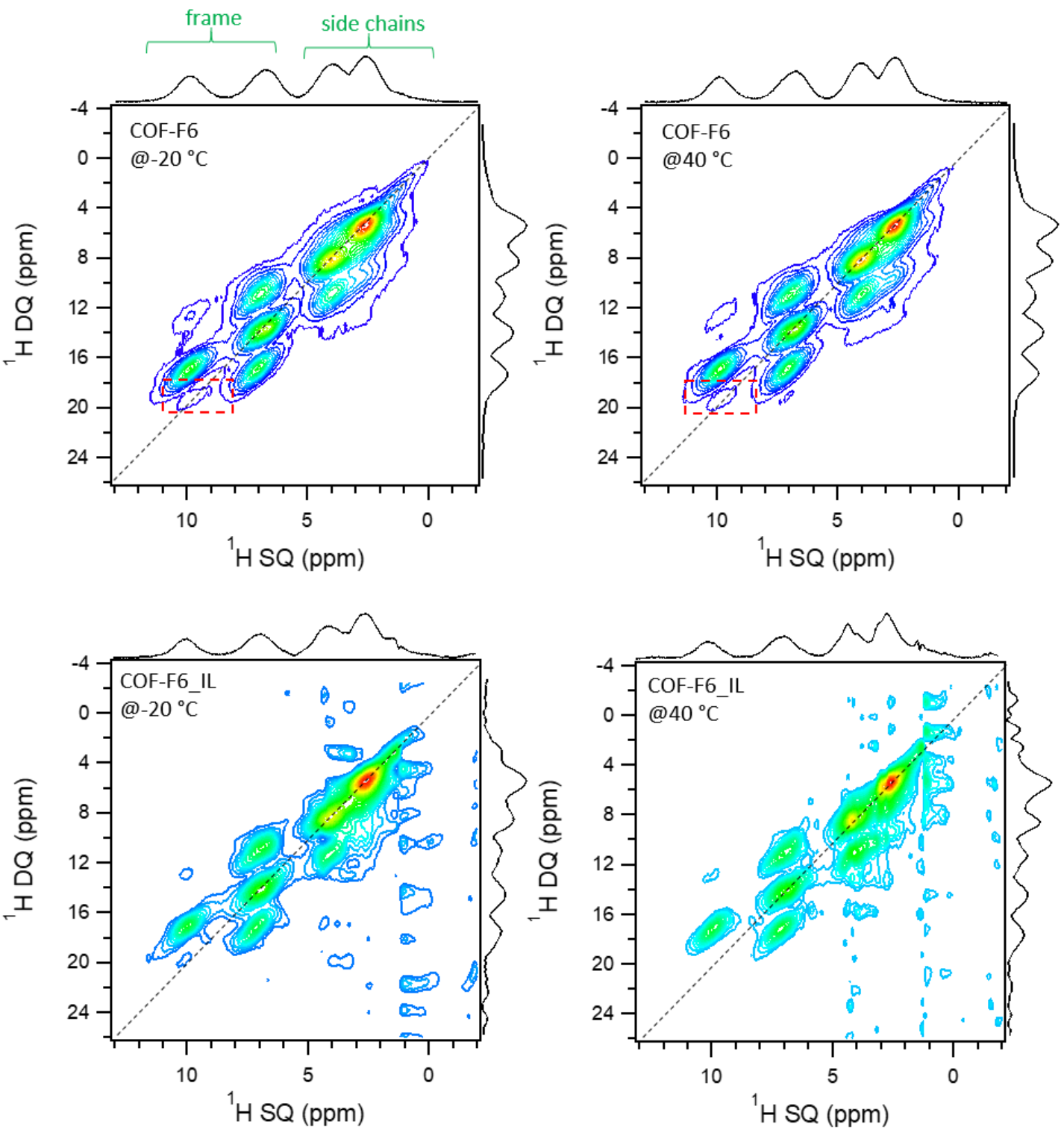

Figure S21 2D ${ }^{1} \mathrm{H}$ DQ/SQ spectra of COF-F6 and F6-IL at -20 and $40{ }^{\circ} \mathrm{C}$.

*Note: The almost identical 2D ${ }^{1} \mathrm{H}$ DQ-SQ spectra of COF-F6 and F6-IL also confirm the COFF6 structure. In the case of COF-F6, the correlations indicated by the red boxes are due to inter-frame interaction $(\mathrm{NH})$, which indicates the distance between the frames below $\sim 3.5 \AA$. 


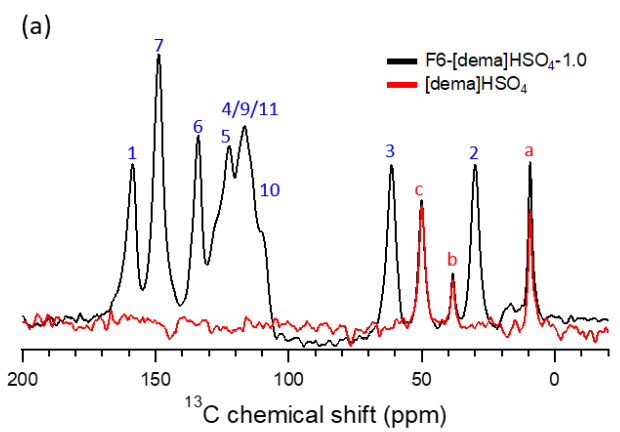

(b)
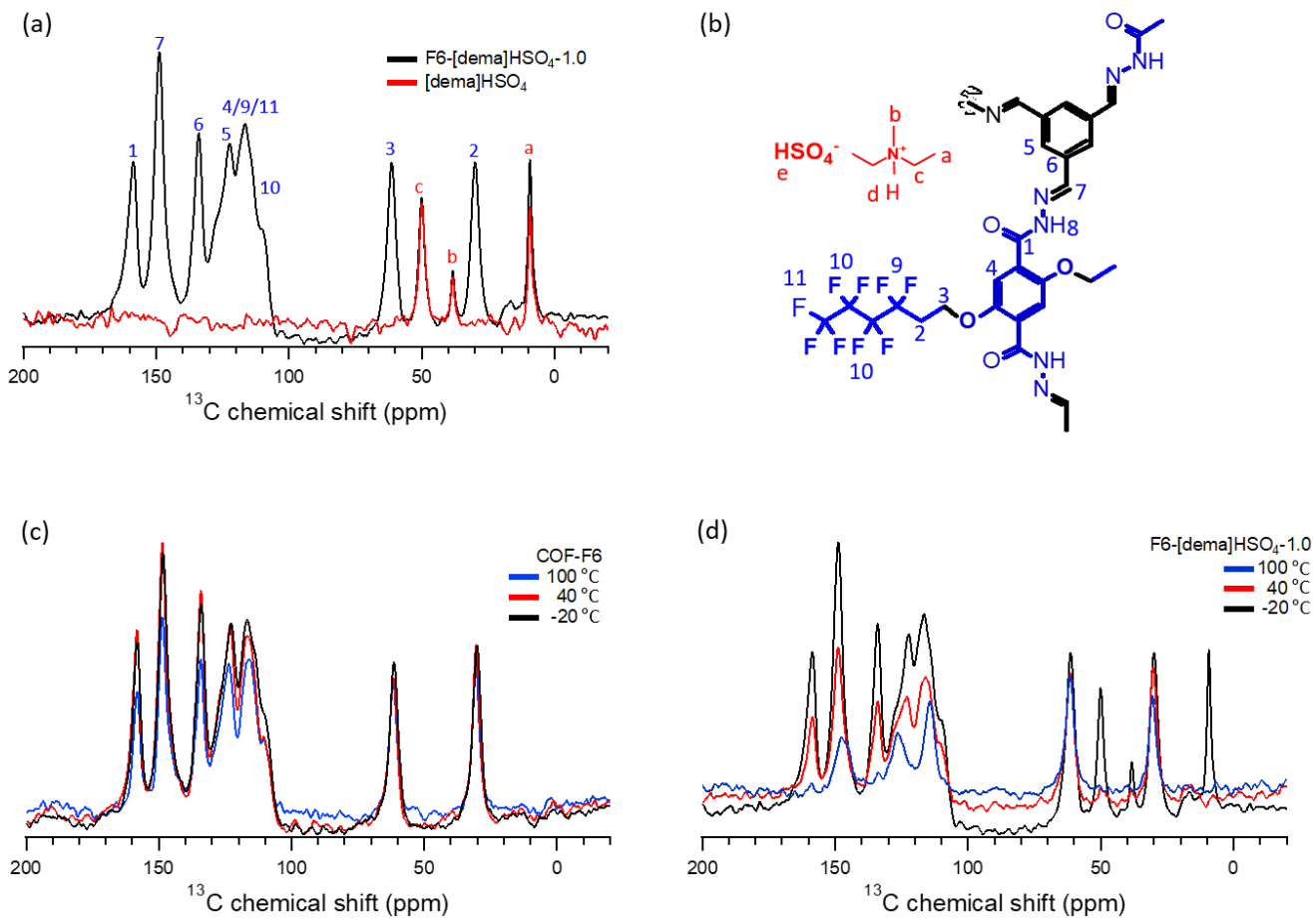

Figure S22 1D $\left\{{ }^{1} \mathrm{H}\right\}{ }^{13} \mathrm{C}$ CPMAS spectra of F6-[dema]HSO 4 -1.0 and IL (a), measured at $-20{ }^{\circ} \mathrm{C}$ and contact time during cross polarization of $2 \mathrm{~ms}$, with the ${ }^{13} \mathrm{C}$ assignment of the structural formula of F6-[dema]HSO4 (b). 1D $\left\{{ }^{1} \mathrm{H}\right\}{ }^{13} \mathrm{C}$ CPMAS spectra of COFF6 (c) and F6-[dema]HSO4-1.0 (d) measured at different temperatures. The contact time of 1D $\left\{{ }^{1} \mathrm{H}\right\}{ }^{13} \mathrm{C}$ CPMAS spectra of F6-[dema]HSO $4-1.0$ measured at $100{ }^{\circ} \mathrm{C}$ set up 0.5 us and the contact time of the other spectra was $2 \mathrm{~ms}$ during cross polarization.

"Note: The ${ }^{13} \mathrm{C}$ signal intensity of COF-F6 is almost similar as a function of temperature while the ${ }^{13} \mathrm{C}$ signal intensity of F6-IL decreased due to the increased mobility at high temperature. Especially, the IL signals at ${ }^{13} \mathrm{C}=50.2,38.4$, and $9.3 \mathrm{ppm}$ disappeared at 40 and $100{ }^{\circ} \mathrm{C}$ due to the increased molecular motion as the temperature increases. The disappearance of quaternary carbon signals of COF-F6 frameworks at ${ }^{13} \mathrm{C}=158.7$ and 134.0 ppm also indicate wobbling of COF framework at $100{ }^{\circ} \mathrm{C}$. 

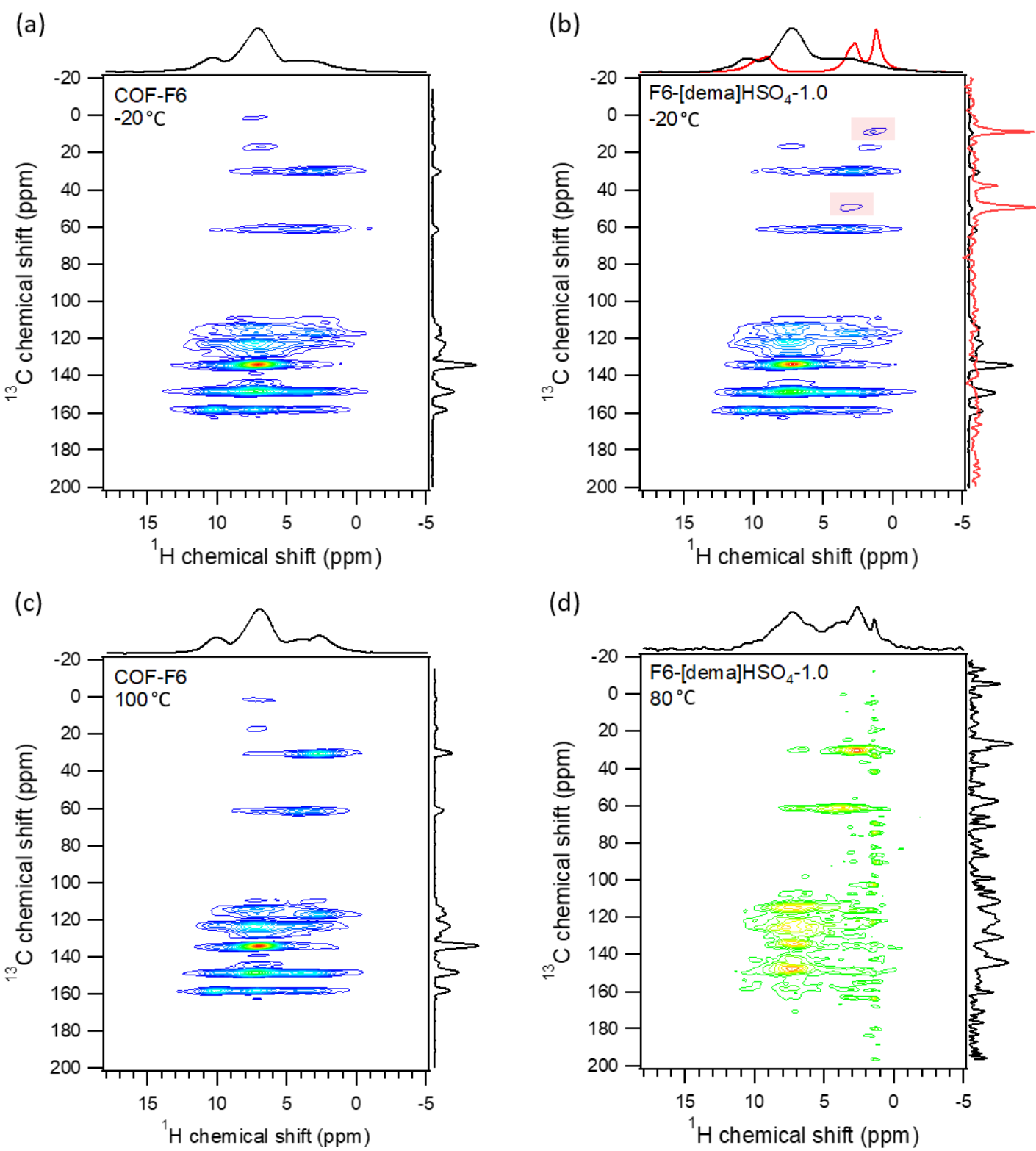

Figure S23 Comparison of ${ }^{1} \mathrm{H}-{ }^{13} \mathrm{C}$ HETCOR spectra of COF-F6 (a) and F6-IL (b) measured at low temperature $\left(-20{ }^{\circ} \mathrm{C}\right)$ and contact time of $2 \mathrm{~ms}$. Comparison of ${ }^{1} \mathrm{H}-{ }^{13} \mathrm{C}$ HETCOR spectra of COF-F6 (c) and F6-IL (d) measured at high temperature (80 and $100{ }^{\circ} \mathrm{C}$ ) and contact time of $2 \mathrm{~ms}$. In (b), the red spectra are ${ }^{1} \mathrm{H}$ single pulse and $\left\{{ }^{1} \mathrm{H}\right\}{ }^{13} \mathrm{C}$ CPMAS of IL.

*Note: The comparison of ${ }^{1} \mathrm{H}_{-}{ }^{13} \mathrm{C}$ spectra of COF-F6 and F6-IL shows almost identical spectra at low and high temperature, which means the frameworks are stable at low and high temperature. The signals enclosed the red boxes in the $2 \mathrm{D}{ }^{1} \mathrm{H}-{ }^{13} \mathrm{C}$ HETCOR spectrum of F6IL at $-20^{\circ} \mathrm{C}$ would be intra-interaction of $\mathbf{I L}$. 

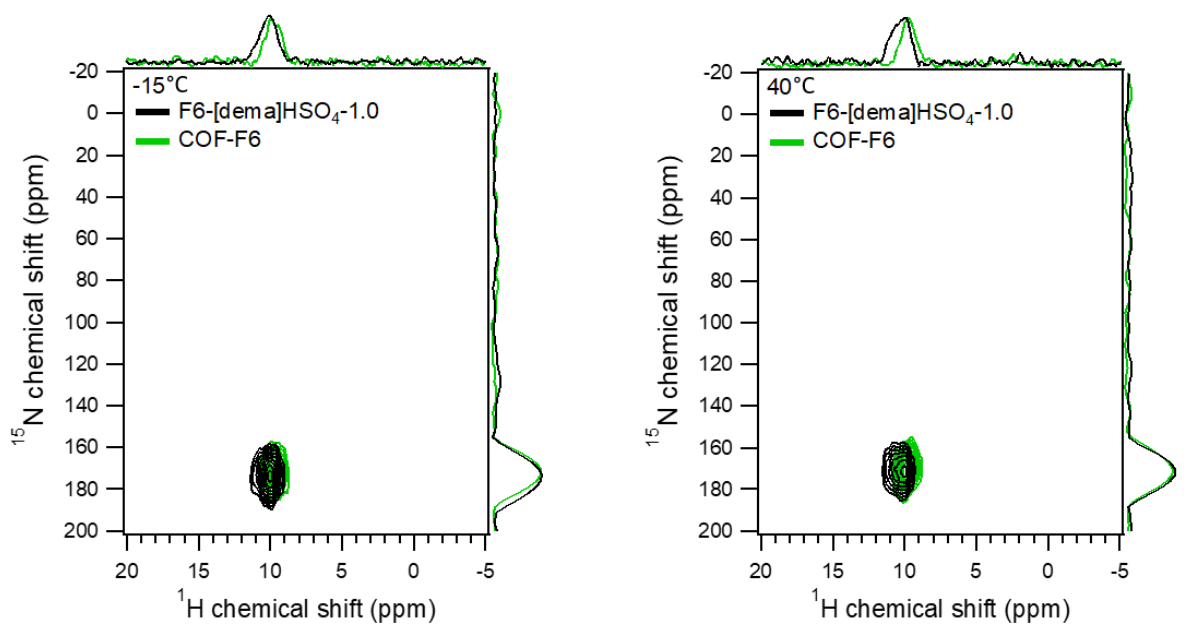

Figure S24. ${ }^{1} \mathbf{H}-{ }^{15} \mathbf{N}$ HETCOR spectra of COF-F6 and F6-IL were measured at -15 and $40{ }^{\circ} \mathrm{C}$ and contact time of $2 \mathrm{~ms}$.

"Note: IL insertion introduces small ${ }^{1} \mathrm{H}$ downfield shift (black spectra, $\sim 0.3 \mathrm{ppm}$ shift comparing the ${ }^{1} \mathrm{H}$ signal of $\mathrm{NH}$ for COF-F6, which may be due to weak hydrogen bonding between IL and framework.

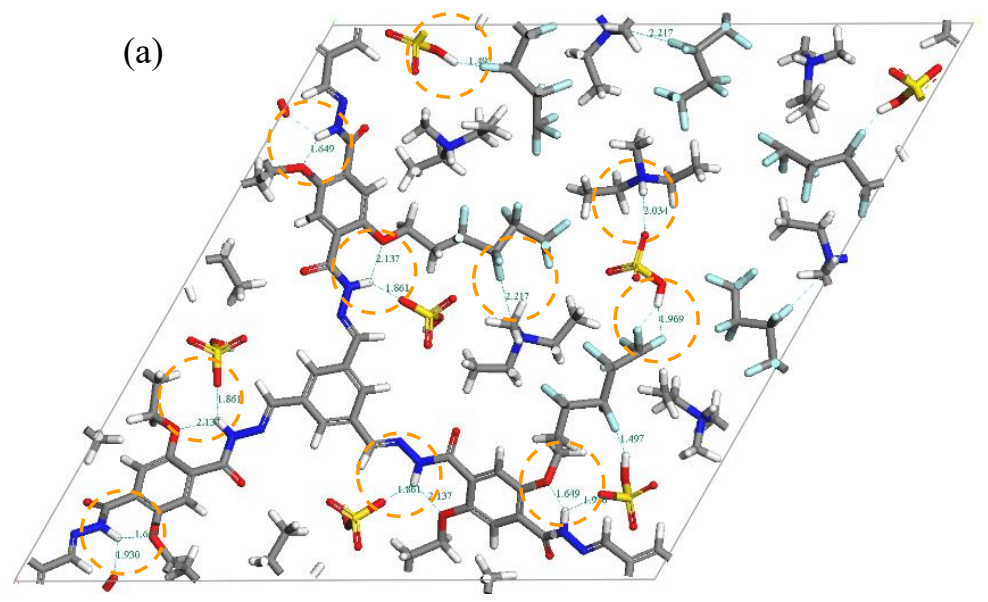

(b)

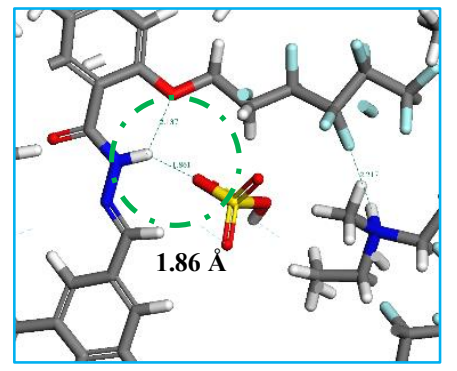

(c)

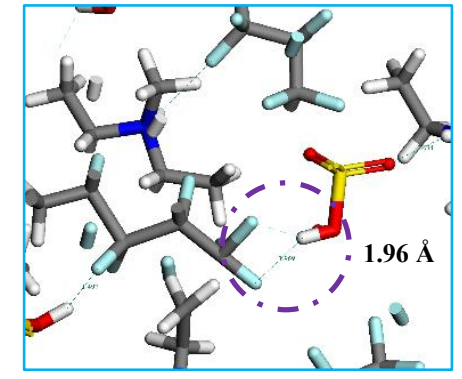

(d)

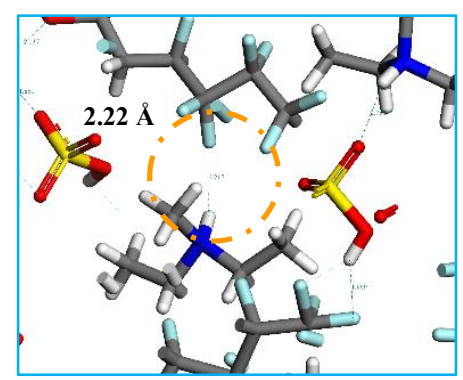

Figure S25. (a) H-bondings in COF-F6, which exists between (b) hydrazone and $\mathrm{HSO}_{4}{ }^{-1}(\mathrm{~N}-\mathrm{H} \cdots \mathrm{O}),(\mathrm{c})(\mathrm{O}-\mathrm{H} \cdots \mathrm{F})$ and $(\mathrm{d})[$ dema] and $\mathrm{CF}$ chains $(\mathrm{N}-\mathrm{H} \cdots \mathrm{F})$

*Note: The geometrical structure of [dema] $\mathrm{HSO}_{4}$ molecule was optimized by Gaussian 
09 (E.01) program with b3lyp/6-31g(d) level. Simulation of hydrogen bonds between the COF-F6 framework and the [dema]HSO ${ }_{4}$ molecules were done by molecular dynamics method, initially several [dema] $\mathrm{HSO}_{4}$ molecules were put into the pores of COF-F6, subsequently followed by geometry optimization performed in Material Studio utilizing Forcite module in which COMPASSII were adopted as the forcefield and Rappe and Goddard's QEq scheme were taken into account. The results clearly demonstrated that $\mathrm{H}$ bonding exists between hydrazone and $\mathrm{HSO}_{4}^{-1}\left(\mathrm{~N}-\mathrm{H}^{\cdots} \mathrm{O}\right.$, Fig S25b), CF chains and $\mathrm{HSO}_{4}{ }^{-1}$ $\left(\mathrm{O}-\mathrm{H}^{\cdots} \mathrm{F}\right.$, Fig S25c) and [dema] and CF chains (N-H $\cdots \mathrm{F}$, Fig S25d).
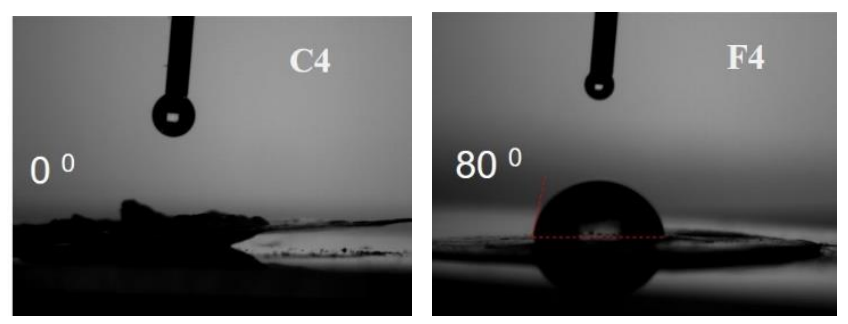

Figure S26. Water contact angles for COF-C4/F4.

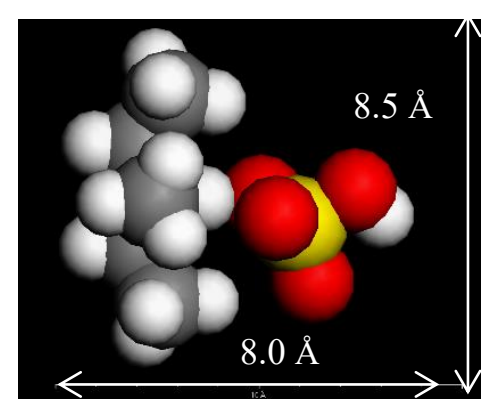

Figure S27. Size of [dema] $\mathrm{HSO}_{4}$ based on the calculated structure optimized by Gaussian 09 (E.01) program with b3lyp/6-31g(d) level.
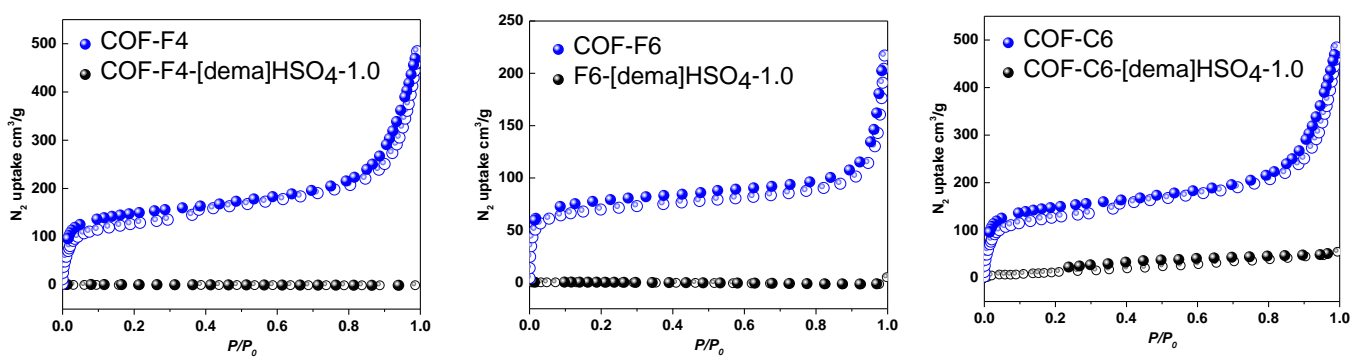

Figure S28. $\mathrm{N}_{2}$ adsorption-desorption measurements for IL-doped COF. 


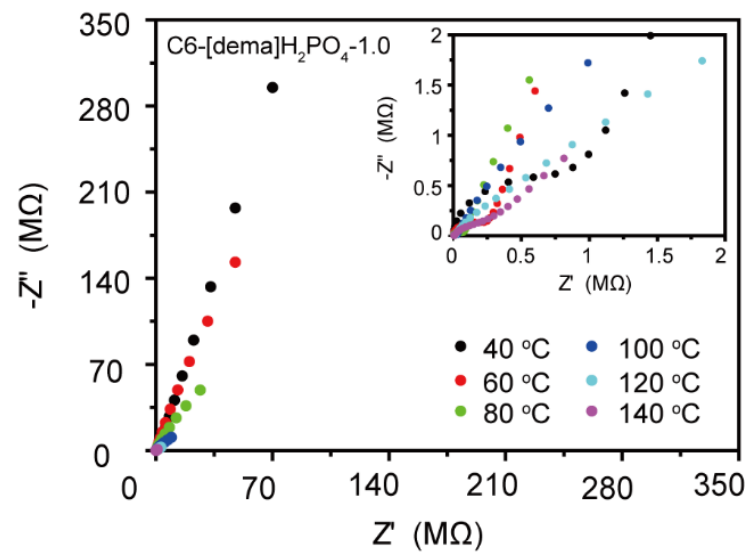

Figure S29. Nyquist plots of C6-[dema] $\mathrm{H}_{2} \mathrm{PO}_{4}-1.0$ measured at different temperatures under anhydrous conditions.

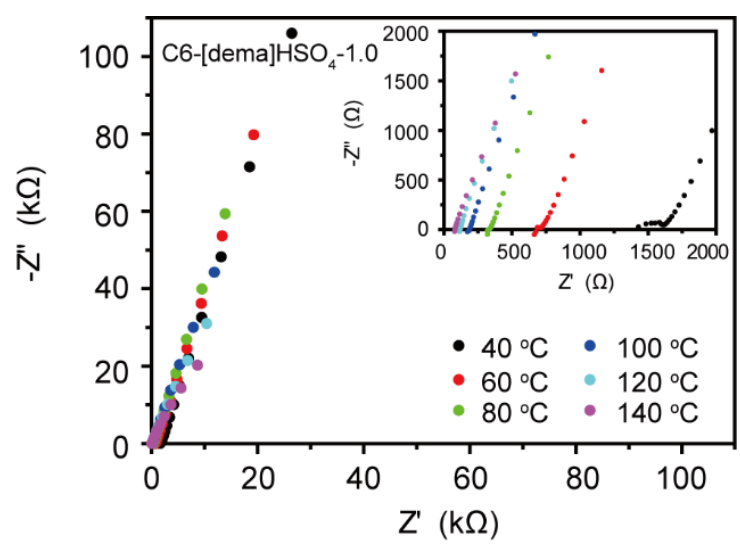

Figure S30. Nyquist plots of C6-[dema]HSO4-1.0 measured at different temperatures under anhydrous conditions.

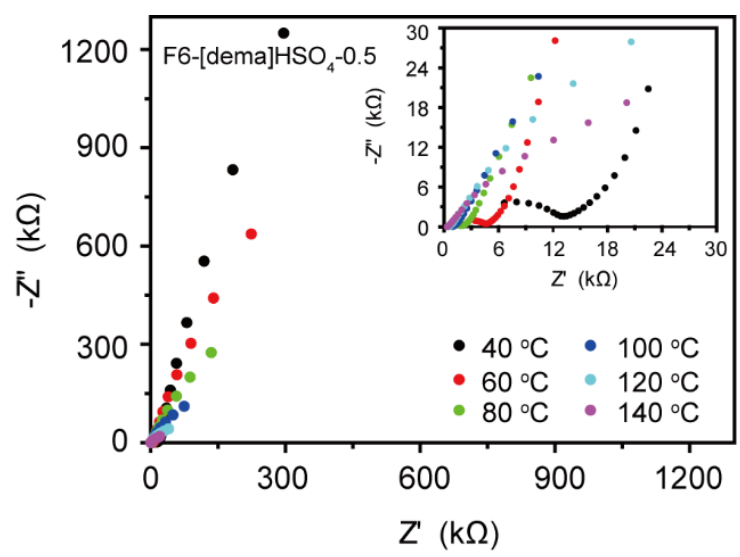

Figure S31 Nyquist plots of F6-[dema]HSO4-0.5 measured at different temperatures under anhydrous conditions. 


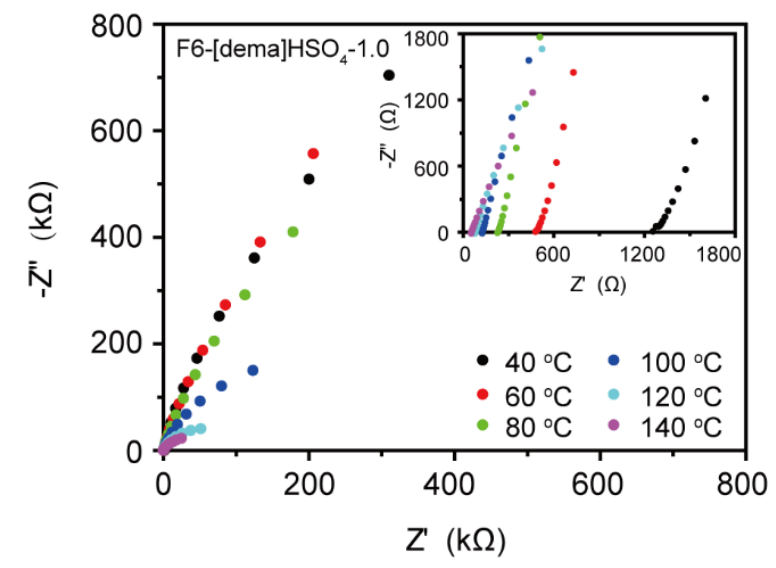

Figure S32. Nyquist plots of F6-[dema]HSO4-1.0 measured at different temperatures under anhydrous conditions.

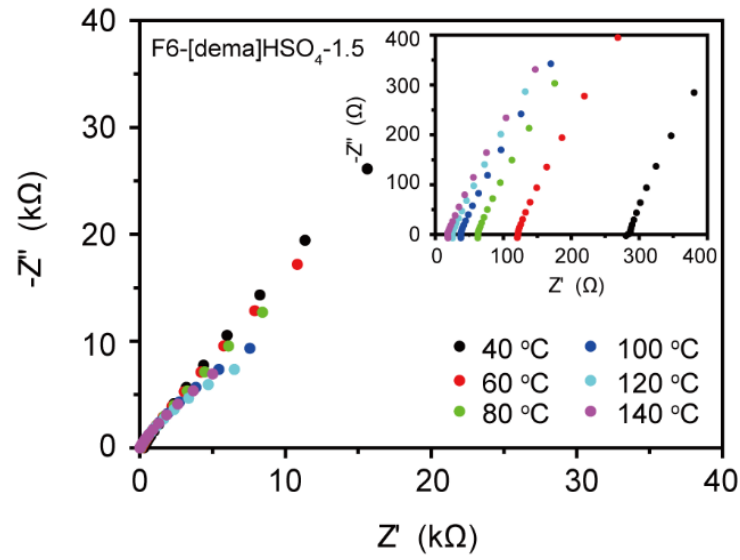

Figure S33. Nyquist plots of F6-[dema]HSO4-1.5 measured at different temperatures under anhydrous conditions. 


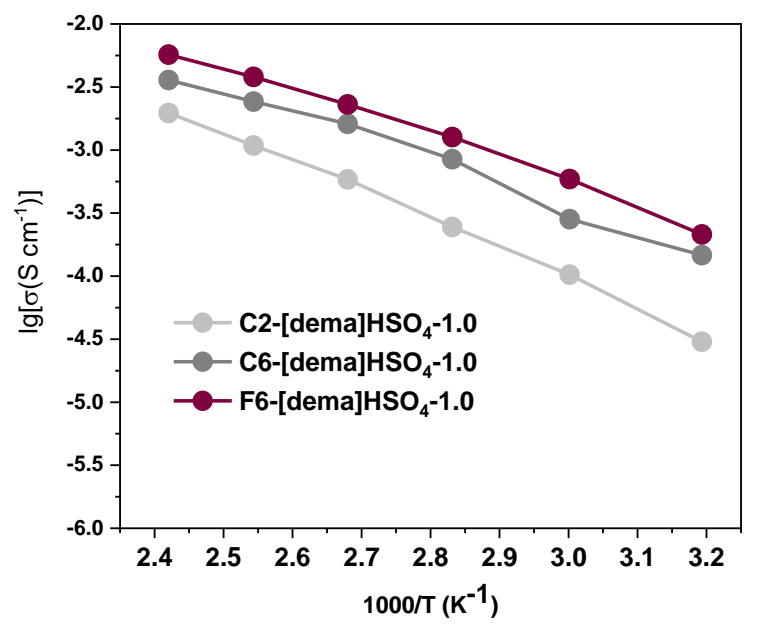

Figure S34. Temperature profiles of proton conductivities of C2/C6/F6-[dema]HSO41.0

Table S4. Comparison of proton conductivities in anhydrous conditions.

\begin{tabular}{|c|c|c|c|}
\hline Material & $\begin{array}{c}\text { Proton conductivity } \\
\left(\mathrm{S} \mathrm{cm}^{-1}\right)\end{array}$ & $\begin{array}{c}\mathrm{T} \\
\left({ }^{\circ} \mathrm{C}\right)\end{array}$ & References. \\
\hline $\begin{array}{c}\mathrm{H}_{3} \mathrm{PO}_{4} @ \text { @PB-DABI-COF (66 } \\
\text { wt\%) }\end{array}$ & $1.52 \times 10^{-1}$ & 160 & $\begin{array}{l}\text { Angew. Chem. Int. Ed. 2021, 60, } \\
12918\end{array}$ \\
\hline CMP-F6 & $4.39 \times 10^{-3}$ & 120 & $\begin{array}{c}\text { ACS Appl. Mater. Interfaces. 2021, } \\
\text { 13, } 15536\end{array}$ \\
\hline $\mathrm{H}_{3} \mathrm{PO}_{4} @ \mathrm{TPB}-\mathrm{DMeTPCOF}$ & $1.91 \times 10^{-1}$ & 160 & Nat. Commun. 2020, 11, 1981 \\
\hline $\mathrm{H}_{3} \mathrm{PO}_{4} @ \mathrm{Tp}-\mathrm{Azo}-\mathrm{COF}$ & $6.70 \times 10^{-5}$ & 67 & JACS. 2014, 136, 6570 \\
\hline $\mathrm{H}_{3} \mathrm{PO}_{4} @$ COF-F6 & $4.2 \times 10^{-2}$ & 140 & J. Am. Chem. Soc. 2020, 142, 14357 \\
\hline $\mathrm{H}_{3} \mathrm{PO}_{4} @ \mathrm{~EB}-\mathrm{COF}$ & $2.77 \times 10^{-2}$ & 140 & J. Mater. Chem. A. 2020, 8, 13702 \\
\hline $\mathrm{H}_{3} \mathrm{PO}_{4} @ \mathrm{TpBpy}-\mathrm{MC}$ & $2.50 \times 10^{-3}$ & 120 & J. Mater. Chem. A. 2016, 4, 2682 \\
\hline $\begin{array}{l}\text { Phytic acid@ } \mathrm{TpPa}-\left(\mathrm{SO}_{3} \mathrm{H}-\right. \\
\text { Py) }\end{array}$ & $5.00 \times 10^{-4}$ & 120 & Chem. Mater. 2016, 28, 1489 \\
\hline Im@TPB-DMTP-COF & $4.37 \times 10^{-3}$ & 130 & Nat. Mater. 2016, 15, 722 \\
\hline Tri@TPB-DMTP-COF & $1.10 \times 10^{-3}$ & 130 & Nat. Mater. 2016, 15, 722 \\
\hline F6-[dema]HSO ${ }_{4}-1.5$ & $1.33 \times 10^{-2}$ & 140 & This work \\
\hline
\end{tabular}

\section{Reference:}

[1] Nakamoto, H.; Watanabe, M. Bronsted Acid-Base Ionic Liquids for Fuel Cell Electrolytes. Chem Commun (Camb). 2007, 24, 2539-2541.

[2] Uribe-Romo, F. J.; Doonan, C. J.; Furukawa, H.; Oisaki, K.; Yaghi, O. M. Crystalline Covalent Organic Frameworks with Hydrazone Linkages. J. Am. Chem. Soc. 2011, 133, 11478-11481. 